\title{
Conservation of tRNA and rRNA 5- methylcytosine in the kingdom Plantae
}

\author{
Alice Louise Burgess ${ }^{1,2+}$, Rakesh David ${ }^{1,2+}$ and lain Robert Searle ${ }^{1,2,3^{*}}$
}

\begin{abstract}
Background: Post-transcriptional methylation of RNA cytosine residues to 5-methylcytosine $\left(\mathrm{m}^{5} \mathrm{C}\right)$ is an important modification that regulates RNA metabolism and occurs in both eukaryotes and prokaryotes. Yet, to date, no transcriptome-wide identification of $\mathrm{m}^{5} \mathrm{C}$ sites has been undertaken in plants. Plants provide a unique comparative system for investigating the origin and evolution of $\mathrm{m}^{5} \mathrm{C}$ as they contain three different genomes, the nucleus, mitochondria and chloroplast. Here we use bisulfite conversion of RNA combined with high-throughput Illumina sequencing (RBS-seq) to identify single-nucleotide resolution of $\mathrm{m}^{5} \mathrm{C}$ sites in non-coding ribosomal RNAs and transfer RNAs of all three sub-cellular transcriptomes across six diverse species that included, the single-celled algae Nannochloropsis oculata, the macro algae Caulerpa taxifolia and multi-cellular higher plants Arabidopsis thaliana, Brassica rapa, Triticum durum and Ginkgo biloba.

Results: Using the plant model Arabidopsis thaliana, we identified a total of 39 highly methylated $\mathrm{m}^{5} \mathrm{C}$ sites in predicted structural positions of nuclear tRNAs and $7 \mathrm{~m}^{5} \mathrm{C}$ sites in rRNAs from nuclear, chloroplast and mitochondrial transcriptomes. Both the nucleotide position and percent methylation of tRNAs and rRNAs $\mathrm{m}^{5} \mathrm{C}$ sites were conserved across all species analysed, from single celled algae N. oculata to multicellular plants. Interestingly the mitochondrial and chloroplast encoded tRNAs were devoid of $\mathrm{m}^{5} \mathrm{C}$ in A. thaliana and this is generally conserved across Plantae. This suggests independent evolution of organelle methylation in animals and plants, as animal mitochondrial tRNAs have $\mathrm{m}^{5} \mathrm{C}$ sites. Here we characterize 5 members of the RNA 5-methylcytosine family in Arabidopsis and extend the functional characterization of TRDMT1 and NOP2A/OLI2. We demonstrate that nuclear IRNA methylation requires two evolutionarily conserved methyltransferases, TRDMT1 and TRM4B. trdmt1 trm4b double mutants are hypersensitive to the antibiotic hygromycin $B$, demonstrating the function of tRNA methylation in regulating translation. Additionally we demonstrate that nuclear large subunit $25 \mathrm{~S}$ rRNA methylation requires the conserved RNA methyltransferase NSUN5. Our results also suggest functional redundancy of at least two of the NOP2 paralogs in Arabidopsis.
\end{abstract}

Conclusions: Our data demonstrates widespread occurrence and conservation of non-coding RNA methylation in the kingdom Plantae, suggesting important and highly conserved roles of this post-transcriptional modification.

Keywords: RNA 5-methylcytosine, Non-coding RNA, Ribosomal RNA (rRNA), Transfer RNA (tRNA), Arabidopsis thaliana, TRDMT1, DNMT2, TRM4, NOP2, NSUN5

\footnotetext{
* Correspondence: lain.Searle@adelaide.edu.au

${ }^{\dagger}$ Equal contributors

${ }^{1}$ School of Biological Sciences, The University of Adelaide, Adelaide, South

Australia 5005, Australia

${ }^{2}$ School of Agriculture, Food and Wine, The Waite Research Institute, The

University of Adelaide, Adelaide, South Australia 5005, Australia

Full list of author information is available at the end of the article
} 


\section{Background}

5-methylcytosine $\left(\mathrm{m}^{5} \mathrm{C}\right)$ is a modification that occurs both on DNA and RNA. In DNA, $\mathrm{m}^{5} \mathrm{C}$ has been extensively studied due to its ease of detection and functional roles of DNA methylation in eukaryotes have been demonstrated for transcriptional silencing of transposons and transgenes, genomic imprinting and $\mathrm{X}$ chromosome inactivation (reviewed in [1]). While DNA appears to be devoid of other modifications [1], RNA has over 100 different modifications that have been identified in different species across all three domains of life [2-4]. Transfer RNAs (tRNAs) are heavily decorated with modifications that have been shown to stabilize secondary structure, affect codon identification and tRNA aminoacylation [5-8]. Of these modifications, $\mathrm{m}^{5} \mathrm{C}$ sites in tRNAs are commonly identified in the variable region and anticodon loop. In response to oxidative stress, $\mathrm{m}^{5} \mathrm{C}$ has been demonstrated to be dynamically modulated in yeast $[9,10]$ and $\mathrm{m}^{5} \mathrm{C}$ plays an important role in regulating tRNA stability and translation in mice under controlled conditions [11]. Furthermore, $\mathrm{m}^{5} \mathrm{C}$ is required for tRNA stability under heat stress and oxidative stress conditions in fruit flies [12]. In ribosomal RNAs (rRNA), $\mathrm{m}^{5} \mathrm{C}$ sites are thought to play a role in translation, rRNA processing and structure [13-15].

In eukaryotes, transfer RNA $\mathrm{m}^{5} \mathrm{C}$ methylation is catalysed by two RNA methyltransferases (RMTases); the first class of RMTase is known as tRNA specific methytransferase 4 (TRM4) or NOP2/Sun domain protein 2 (NSUN2), in yeast and animals respectively $[11,16,17]$. NSUN2 mutations in humans are linked to inherited intellectual disability and this is thought to be mediated by increased cleavage of tRNAs by the ribonuclease angiogenin [18-22]. In mice, nsun 2 mutants are smaller and have reduced male fertility and have revealed a role in stem cell self-renewal and differentiation [23, 24]. Using phylogenetic analysis, two putative TRM4/NSUN2 paralogs, TRM4A and TRM4B, were identified in the Arabidopsis genome $[25,26]$, however these genes have not been characterized in plants. The second class of eukaryotic RMTase; Transfer RNA aspartic acid methyltransferase 1 (TRDMT1), also known as DNA methyltransferase 2 (DNMT2), has been shown to methylate tRNAs in Drosophila, Arabidopsis and Homo sapiens. In plants, only one $\mathrm{m}^{5} \mathrm{C}$ site in tRNA ${ }^{\text {Asp(GTC) }}$ at position C38 has been shown to be methylated by TRDMT1 [27]. While Drosophila, and Arabidopsis trdmt1 mutants appear wild type under standard laboratory conditions, zebrafish deficient in TRDMT1 have reduced body size and impaired differentiation of specific tissues [27, 28]. In nuclear encoded eukaryotic tRNAs, $\mathrm{m}^{5} \mathrm{C}$ methylation has been commonly reported at six cytosine positions; C34, C38, C48, C49, C50 and C72 [2, 3, 18, 29-31]. Methylation has also been discovered on mitochondrial encoded tRNAs in humans and cows on several tRNAs at positions C48, C49 and C72 [29, 32]. However, the methylation status of chloroplast encoded tRNAs and rRNAs has not been previously reported.

Like tRNAs, ribosomal RNAs are highly conserved and have important roles in translation. The ribosome consists of two subunits, the large subunit (LSU) and the small subunit (SSU). The LSU is composed of three rRNA species in eukaryotes, and generally two rRNA species in prokaryotes, while the SSU contains only one rRNA species in both prokaryotes and eukaryotes [33-35]. The rRNA sequences are conserved, although the names of rRNA species are often not. Whereas rRNA methylation has not been investigated in plants, the location and enzymatic requirements of a few $\mathrm{m}^{5} \mathrm{C}$ sites in select organisms has been determined. For example, the human nuclear LSU rRNAs (28S and 5S) are methylated. The 28S rRNA contains two sites at C3782 and C4447 while 5S rRNA is methylated at C92 [30, 31, 36]. The orthologous yeast LSU $25 \mathrm{~S}$ rRNA contains two sites at C2278 and C2870 [13, 15] and E. coli LSU 23S rRNA at C1962 [37] and SSU 16S rRNA at C967 [38] and C1407 [39]. Hamster mitochondrial SSU 13S rRNA also contains one $\mathrm{m}^{5} \mathrm{C}$ site [40], similarly mouse mitochondrial SSU $12 \mathrm{~S}$ rRNA is methylated at position C911 [41]. Two RMTases that have been identified to methylate ribosomal RNA in eukaryotes are NOP2 (nucleolar protein 2) and RCM1 (rRNA cytosine methyltransferase 1). NOP2 methylates position C2870 and RCM1 methylates position C2278 in the LSU 25S rRNA in Saccharomyces [13, 15]. Yeast NOP2 is required for correct rRNA biosynthesis and processing [14] and nop $2 \mathrm{mu}-$ tants are lethal. In contrast, yeast $r \mathrm{~cm} 1$ mutants are viable, however they are hypersensitive to anisomycin and this is thought to be due to structural changes being induced by methylation of rRNA [15]. While there is only one copy of the RCM1 homolog, referred to here as NSUN5 in Arabidopsis, there are three paralogs of NOP2 in the Arabidopsis genome, OLI2 (NOP2A), NOP2B and NOP2C [26]. One of these, NOP2A/OLI2 was identified in a forward genetic screen for genes involved in compensation of cell size [42]. The methylation activity or $\mathrm{m}^{5} \mathrm{C}$ sites mediated by the three Arabidopsis NOP2 paralogs and NSUN5 are unknown. Another RMTase, which is related to the bacterial Fmu rRNA MTase was recently identified in Arabidopsis [43]. Arabidopsis rnmt (RNA methyltransferase) mutants had reduced global RNA methylation, indicating that it may methylate highly abundant rRNA transcripts.

Unlike animals, plant cells contain three evolutionary distinct genomes; nuclear, mitochondrial and chloroplast, thus providing a unique model for investigating $\mathrm{m}^{5} \mathrm{C}$ catalysis and biological function. The mitochondria is a striking example of how a prokaryotic translational machinery has adapted to input from eukaryotic translational machinery as nuclear, eukaryotic tRNAs are required to be imported 
into the mitochondria, as the mitochondria no longer has a full complement of tRNAs [44, 45]. tRNA sequences present in plants are dynamic, as there are multiple copies of each tRNA isodecoder and these can be lost within a genome or transferred from the chloroplast and mitochondrial genomes to the nucleus [46]. This gives rise to incidents where a nuclear encoded tRNA has an organelle-like sequence. It is unknown whether these "transferred" tRNAs are expressed after integration into a new genome as a systematic analysis of tRNA expression in plants is yet to be undertaken [47-49].

In this study, we describe single nucleotide resolution of post-transcriptionally modified cytosine residues in plant rRNA and tRNAs by combining RNA bisulfite conversion with second generation Illumina sequencing (RBS-seq). We report the identification of novel modified cytosines in $A$. thaliana nuclear transcribed tRNAs and that these sites are dependent on RMTases TRDMT1 and the previously undescribed Arabidopsis TRM4B. Additionally, we show these modified sites in nuclear tRNAs are conserved through evolution from the single celled algae Nannochloropsis oculata to multicellular higher plants. Interestingly, no $\mathrm{m}^{5} \mathrm{C}$ sites were detected in Arabidopsis chloroplast or mitochondrial tRNAs, which is in contrast to animal mitochondrial tRNAs. The function of tRNA methylation in regulating translation is demonstrated, as trdmt1 trm $4 b$ double mutants are hypersensitive to the antibiotic hygromycin B. Furthermore, we identify novel modified cytosines in nuclear, mitochondrial and chloroplast rRNAs. In Arabidopsis nuclear LSU $25 \mathrm{~S}$ rRNA, $\mathrm{m}^{5} \mathrm{C}$ at $\mathrm{C} 2268$ was dependent on NSUN5, but methylation at C2860 was not found to be dependent on any particular NOP2 ortholog in Arabidopsis. Furthermore, RMTases responsible for methylation of tRNAs were not required for rRNA methylation, and vice versa indicating functional specialization of the RMTase family. These data represent the first high-resolution description of tRNA and rRNA modifications in the plantae kingdom and creates a platform to begin understanding the function, significance and evolution of non-coding RNA methylation.

\section{Results}

\section{Detection and enrichment of transcribed tRNAs in} Arabidopsis thaliana

To identify transcribed tRNAs in A. thaliana we implemented a two-step approach. First, a tRNA isodecoder consensus list was constructed to facilitate expression analysis and second, a tRNA enrichment protocol combined with Illumina deep-sequencing was developed similar to those recently described [50]. The tRNA isodecoder consensus approach was undertaken as there are over 640 predicted tRNA genes in $A$. thaliana, originating from the nuclear, mitochondrial and chloroplast genomes often with multiple identical isodecoder sequences that makes assigning IIlumina sequences to individual transcribed tRNA loci challenging. Using this consensus approach, the predicted $A$. thaliana tRNAs were resolved into 100 reference consensus sequences (Additional file 1: Table S1).

To identify transcribed tRNAs, we initially used total RNA to construct an Illumina library, deep-sequenced the library and aligned the sequenced reads to our tRNA consensus list. Only $0.0007 \%$ of sequence reads aligned to tRNAs using this traditional approach. Therefore, we developed a method for tRNA enrichment prior to Illumina sequencing similar to those recently described (see Methods). Briefly, after separation of total RNA on a polyacrylamide gel, a region corresponding to the tRNAs was excised, RNA purified and then either bisulfite treated or directly used as template in library construction. Using this enrichment method, a nearly 20,000-fold increase in the sequence reads aligning to tRNAs was observed, when compared to using total RNA (Fig. 1a). Expression of 56 out of 100 isodecoder consensus sequences from all three genomes, nuclear, chloroplast and mitochondrial was observed using our RBS-seq data. Of these, seven tRNA sequences were ambiguously aligning with two or more genomes (Fig. 1b). A wide-range of tRNA transcript abundances were observed from our RNA-seq data, with chloroplast and mitochondrial derived tRNAs having the highest abundance (Fig. 1c). This is most likely a reflection of the high copy number of plastid and mitochondrial organelles per mesophyll cell.

\section{RBS-seq analysis to identify 5-methylcytosine $\left(\mathrm{m}^{5} \mathrm{C}\right)$ sites in tRNAs of $A$. thaliana}

To identify $\mathrm{m}^{5} \mathrm{C}$ sites in tRNAs at single-nucleotide resolution, we performed bisulfite (BS) conversion on enriched tRNAs from wild type Arabidopsis that were combined with an in vitro transcribed Renilla Luciferase (R-Luc) mRNA BS conversion control lacking $\mathrm{m}^{5} \mathrm{C}$. Complete BS conversion of R-Luc control results in no cytosines and serves as an important internal control. After BS conversion, Illumina libraries were constructed, deep-sequenced and aligned to in silico BS converted, cytosine to thymine, endogenous Arabidopsis tRNA consensus sequences and the R-Luc control. For a BS converted sample to pass our quality control standards, the R-Luc control required a minimum of $98 \%$ conversion across the 178 cytosines present in the R-Luc mRNA BS conversion control (Additional file 1: Figure S1A). After passing R-Luc quality control, we then determined the global endogenous cytosine abundance. In all stranded RBS-seq libraries, global endogenous cytosine abundance was less than $\sim 1 \%$ compared to $\sim 22 \%$ for non-BS treated RNA-seq samples (Additional file 1: Figure S1B, S1C). Together these results demonstrated that bisulfite conversion of RNA cytosines was highly efficient using our method. 
A

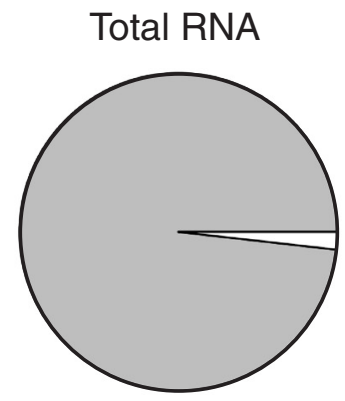

\section{Gel purified RNA}

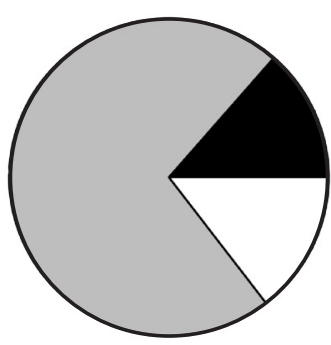

B

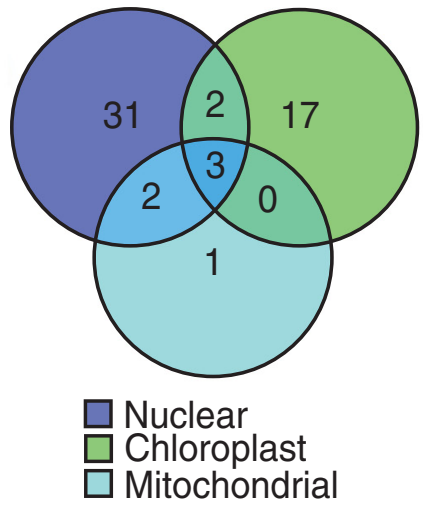

C

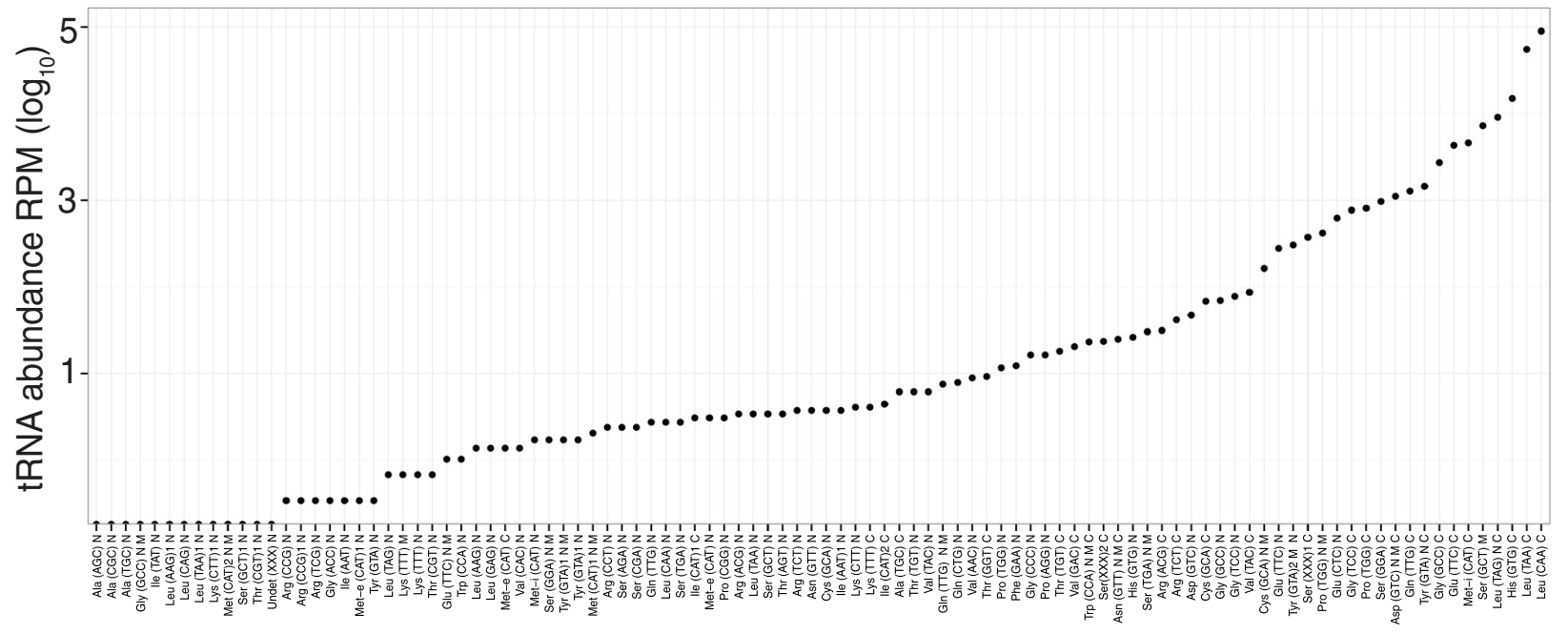

Fig. 1 Efficient detection of Arabidopsis tRNAs by polyacrylamide gel purification and RNA-seq. a Comparison of Illumina sequencing reads from either total RNA or gel purified RNA shows an increase in reads mapping to tRNAs from 0.0007 to $13.58 \%$, respectively. Data from one representative biological replicate is shown. $\mathbf{b}$ Venn diagram showing detection of gel purified tRNA consensus sequences from nuclear, chloroplast and mitochondrial genomes. 56 out of 100 known tRNA consensus sequences were identified in our analysis. Overlapping circles indicate tRNAs that may originate from more than one genome ( $n=3$ biological replicates). c Consensus tRNAs display a wide range of expression levels with chloroplast (C) encoded sequences showing the highest expression levels compared to nuclear $(\mathrm{N})$ and mitochondrial $(\mathrm{M})$ sequences (1 replicate). Three of the tRNAs have undetermined anticodon sequences and are shown as (XXX). Minority isodecoders with diverged sequences from the majority isodecoder are designated by the number 1 or 2 after the anticodon. RBS-seq was used for (a) and (b) and RNA-seq was used in (c)

To identify $\mathrm{m}^{5} \mathrm{C}$ sites in nuclear, chloroplast and mitochondrial Arabidopsis tRNAs, we aligned the Illumina RBS-seq reads against an in silico converted tRNA consensus list. In silico conversion involved converting all cytosines to thymines. 5-methylcytosine sites were then identified as cytosines that resist bisulfite conversion. These sites are to be noted as candidate $\mathrm{m}^{5} \mathrm{C}$ sites, as other types of modified cytosine can also be resistant to bisulfite conversion $[29,51]$. We applied a threshold of at least 5 reads aligning to an individual tRNA consensus and a minimum of $20 \%$ methylation. Using these parameters, we identified 24 methylated tRNAs and 32 non-methylated tRNAs out of a total of 56 (Fig. 2a, Additional file 1: Table
S2). Interestingly, only nuclear encoded tRNAs were found to contain $\mathrm{m}^{5} \mathrm{C}$ sites, whereas non-methylated tRNAs were encoded by all three genomes.

Cytosine methylation of Arabidopsis nuclear tRNAs ranged from 23 to $100 \%$, and were consistent between the three biological replicates. $39 \mathrm{~m}^{5} \mathrm{C}$ sites were identified at 5 structural positions and are illustrated on the representative tRNA secondary structure at positions C38, C48, C49, C50 and C72 (Fig. 2b). Methylation at these sites is consistent with observations in other nonplant species [2, 3, 18, 29-31]. Next we examined the pattern of methylation in individual tRNA isodecoders. Seventeen tRNAs were identified with methylation at 
A

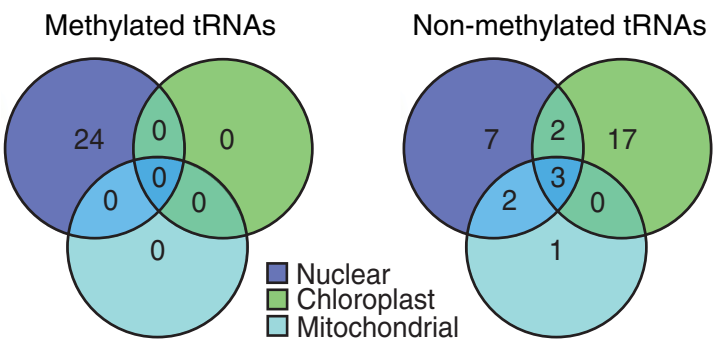

B
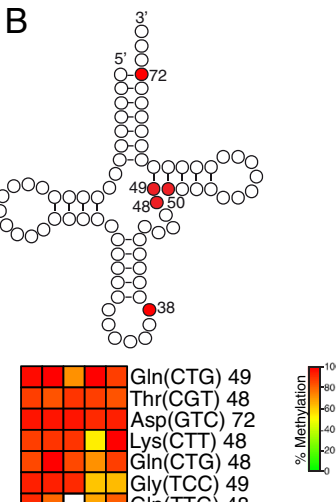

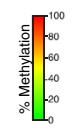

C

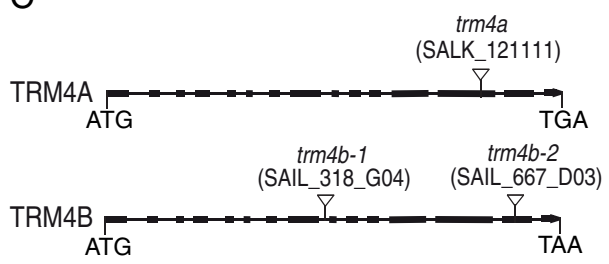

$\mathrm{D}$

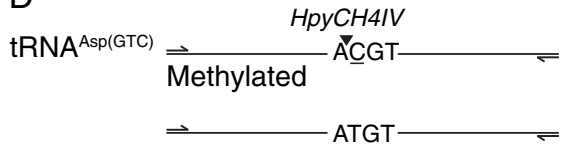

Non-methylated

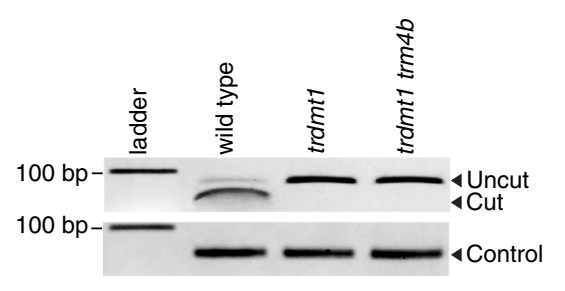

$\mathrm{E}$

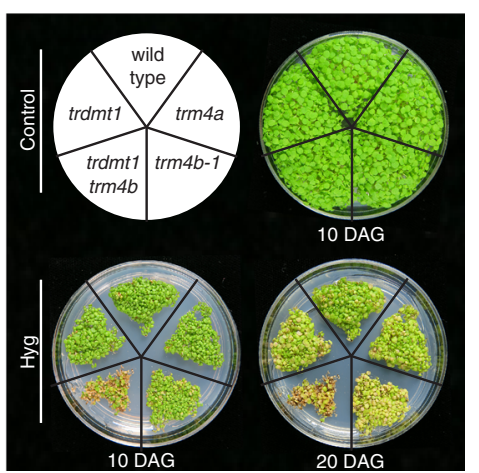

Fig. 2 TRDMT1 and TRM4B methylate Arabidopsis nuclear encoded transfer RNAs. a Genomic origins of methylated and non-methylated tRNAs. Methylated tRNAs were only detected from the nuclear genome (3 biological replicates). b Above: clover-leaf representative secondary structure of tRNA indicating in red, the five cytosine positions methylated in wild type. Below: Heatmap showing percentage methylation of all cytosines detected in nuclear tRNAs of wild type, and mutants trdmt1, trm4a, trm4b-1 and trdmt 1 trm $4 b$ using RBS-seq. Cytosine positions are indicated next to tRNA isodecoders. White boxes represent cytosine positions with coverage less than five reads. (wild type 3 biological replicates, mutants $n=$ 1). $\mathbf{c}$ Genomic structure of trm $4 a$ and trm $4 b$ mutants showing T-DNA insertions (triangles) in exons (filled boxes). $\mathbf{d}$ Analysis of RNA methylation by TRDMT1 at position C38 on BS treated tRNA ${ }^{\text {Asp(GTC) }}$ template. Above: Restriction maps of PCR amplified products showing the expected digest patterns of methylated and non-methylated template. Below: Cleavage of PCR amplified product by HpyCH4IV confirms C38 methylation in wild type as opposed to non-methylated C38 in trdmt1 results in loss of HpyCH4IV restriction site. Loading control is undigested PCR product. e Hygromycin B stress assay. Trdmt1 trm $4 b$ double mutants and to a lesser extent, trm4b-1 mutants display increased sensitivity to hygromycin B (Hyg) at 10 and 20 days after germination (DAG) compared to controls 
only 1 structural position, while the other remaining 7 tRNAs contained $2-5 \mathrm{~m}^{5} \mathrm{C}$ sites per tRNA. The most frequently methylated sites corresponded to structural positions $\mathrm{C} 48, \mathrm{C} 49$ and $\mathrm{C} 50$, indicating that methylation in this region may be important for tRNA structure or stability. tRNA ${ }^{\text {Asp(GTC) }}$ was the most highly methylated tRNA and was the only tRNA containing methylation at all 5 structural positions. The structure of tRNA ${ }^{\text {Asp(GTC) }}$ may require these additional $\mathrm{m}^{5} \mathrm{C}$ sites for greater stability or resistance to cleavage.

\section{Identification of TRM4B and TRDMT1 dependent $\mathrm{m}^{5} \mathrm{C}$ sites in nuclear tRNAs}

To confirm the $\mathrm{m}^{5} \mathrm{C}$ sites in Arabidopsis nuclear tRNAs and determine the RMTases required for methylation, we identified mutants for the predicted Arabidopsis homologs of RMTases TRM4 and TRDMT1 and then performed RBS-seq on libraries enriched for tRNAs.

Two TRM4 paralogs were identified in the Arabidopsis genome [25] and we refer to them as TRM4A and TRM4B. T-DNA mutations in TRM4A or TRM4B were identified and the homozygous mutants characterized by semi-quantitative RT-PCR to demonstrate null expression (Fig. 2c and Additional file 1: Figure S2C) and show mutants are most likely complete loss of function. Mutants trm $4 a$, and the two isolated T-DNA mutants for TRM 4B; trm $4 b-1$ and trm $4 b-2$ were grown on soil and appeared phenotypically similar to wild type like the previously characterized RMTase mutant trdmt1 [27] (Additional file 1: Figure S2A). To test for divergent functions of TRM4A and TR4MB, the $\mathrm{m}^{5} \mathrm{C}$ singlenucleotide profile of tRNAs was determined in the mutants (Fig. 2b). In trm $4 a$, the $\mathrm{m}^{5} \mathrm{C}$ profile was the same as wild type, showing that TRM4A is not required for methylation of any of the detected tRNAs. In contrast for trm $4 b-1$ and trm $4 b-2$, a total of 18 sites had no detectable methylation and 7 sites had reduced methylation when compared to wild type (Fig. 2b and Additional file 1: Figure S3A). The sites that lost methylation or had reduced methylation corresponded to structural positions C48, C49 and C50 which is consistent with animal studies $[2,3,18,29-31]$.

Further investigation of the functional motifs of TRM4A and TRM4B by sequence alignment demonstrated that TRM4A is missing motif I (Additional file 1: Figure S4A). Motif I is essential for methyltransferase activity and is required for AdoMet binding and catalysis [52]. Loss of motif I in TRM4A most likely explains why no reduction in tRNA $\mathrm{m}^{5} \mathrm{C}$ levels was observed in trm4a. However we cannot exclude the possibility that TRM4A has other functional roles. As TRM4B contains all of the predicted motifs required for RMTase activity and there is a reduction in $\mathrm{m}^{5} \mathrm{C}$ tRNA methylation in the trrm $4 b$ mutants, this demonstrated that TRM4B is the functional homolog of TRM4/NSUN2 in Arabidopsis thaliana.

TRDMT1 was previously reported to methylate three tRNAs, tRNA ${ }^{\text {Asp(GTC) }}$, tRNA ${ }^{\text {Gly(GCC) }}$ and tRNA ${ }^{\text {Val(AAC) }}$ at structural position C38, in animals [11, 12, 27, 30] and

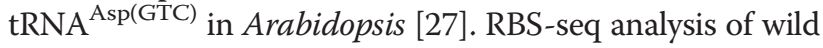
type Arabidopsis and trdmt 1 not only confirmed that TRDMT1 is required for position C38 methylation of tRNA $^{\text {Asp(GTC) }}$ but is also required for C38 methylation of tRNA $^{\text {Gly(CCC) }}$ and tRNA ${ }^{\text {Gly(GCC) }}$ in plants as these sites had no detectable methylation in trdmt1. In contrast to animals, position C38 of tRNA ${ }^{\mathrm{Val}(\mathrm{AAC})}$ is not methylated in Arabidopsis (Additional file 1: Table S2). All other detected tRNAs were not methylated at position C38.

Nine $\mathrm{m}^{5} \mathrm{C}$ sites in nuclear tRNAs did not show a reduction of methylation in trm $4 a-1, \operatorname{trm} 4 b-1$ or trdmt1 single mutants when compared to wild type. These sites occur at structural positions C47, C48, C49 and C72 and are shown clustered together at the top of the heatmap (Fig. 2b). To exclude the possibility of functional redundancy of TRM4B and TRDMT1, we constructed a trdmt1 trm $4 b$ double mutant and then performed RBS-seq. All 9 sites were methylated in the double mutant and therefore we concluded that no functional redundancy of TRM4B and TRDMT1 for methylation of specific cytosine residues occurs in Arabidopsis. We cannot rule out the possibility that these 9 sites are cytosines with other RNA modifications that, like $\mathrm{m}^{5} \mathrm{C}$, are also resistant to bisulfite conversion and therefore are independent of TRM4A, TRM4B, or TRDMT1 methylation.

To further demonstrate the reproducibility of our tRNA methylation data, we developed a rapid PCR-digestion assay to investigate individual $\mathrm{m}^{5} \mathrm{C}$ sites derived from BS treated RNA. Position C38 of tRNA ${ }^{\text {Asp(GTC) }}$ coincides with the restriction enzyme digestion site, ACGT, of HpyCH4IV. Methylation of $\mathrm{C} 38$ protects the site from BS conversion maintaining the HpyCH4IV site in methylated tRNA ${ }^{\text {Asp(GTC) }}$ derived PCR products. Therefore HpyCH4IV only cleaves tRNA $^{\text {Asp(GTC) }}$ PCR products when position C38 is methylated. Methylation of tRNA ${ }^{\text {Asp(GTC) }}$ at position C38 by TRDMT1 was confirmed using the digestion assay on wild type and trdmt1 BS treated RNA (Fig. 2d). As expected, C38 of tRNA ${ }^{\text {Asp(GTC) }}$ is not methylated in trdmt1 or trdmt1 trm $4 b$ double mutants and is not cleaved by HpyCH4IV after BS treatment. The rapid digestion assay confirmed our RBS-seq data.

To test the role of tRNA $\mathrm{m}^{5} \mathrm{C}$ sites in regulating translation, the antibiotic hygromycin $\mathrm{B}$, hereafter described as hygromycin, was used to perturb translation. Hygromycin alters the conformation of the A-site in the ribosome, which increases binding of tRNAs to the A-site, inhibits translocation and reduces translational fidelity [53]. The tRNA RMTases TRDMT1 and TRM4B mutants are expected to be more sensitive to hygromycin, as the loss of 
methylation is predicted to weaken the structural integrity of select tRNAs and increase the ability of hygromycin to bind and 'lock' tRNAs in the A-site, stopping translocation. Therefore we tested this expectation by growing wild type and mutants on control and hygromycin containing plates. Both trm $4 b$ and trdmt 1 trm $4 b$ double mutants displayed increased sensitivity to hygromycin at 10 and 20 days after germination (DAG) when compared to the controls (Fig. 2e). The sensitivity of trm $4 b$ mutants to hygromycin is more apparent at 20 days DAG than at 10 DAG. As a number of tRNAs lose methylation in trm $4 b$ and trdmt 1 trm $4 b$ mutants (Fig. $2 \mathrm{~b}$ ) and previous reports that loss of methylation affects tRNA structure, we attribute the hygromycin sensitivity of the mutants to a modified tRNA structure and the increased interaction between these tRNAs and the A-site of the ribosome reducing translation.

\section{Identification of $\mathrm{m}^{5} \mathrm{C}$ sites in Arabidopsis nuclear, chloroplast and mitochondrial ribosomal RNAs}

To identify $\mathrm{m}^{5} \mathrm{C}$ sites in rRNAs from $A$. thaliana, we first constructed a list of rRNA sequences to represent all rRNAs from nuclear, mitochondrial and chloroplast genomes (Additional file 1: Table S3). Then we in silico bisulfite converted all cytosines to thymines before aligning the RBS-seq data. RBS-seq transcriptome libraries from total RNA were sequenced and efficient bisulfite conversion of cytosine residues was determined as previously described (Additional file 1: Figure S1A, S1B and Methods).

We identified a total of $7 \mathrm{~m}^{5} \mathrm{C}$ sites in the nuclear LSU $25 \mathrm{~S}$ rRNA, chloroplast SSU 16S, LSU 23S and mitochondrial SSU 18 S and LSU $26 \mathrm{~S}$ rRNAs (Fig. 3a, b). This pattern is in contrast to tRNA methylation, which was only detected on nuclear tRNAs (Fig. 2a). Each methylated rRNA contained one $\mathrm{m}^{5} \mathrm{C}$ site except for the nuclear LSU $25 \mathrm{~S}$ and chloroplast LSU 23S rRNAs that each contained two $\mathrm{m}^{5} \mathrm{C}$ sites (Fig. $3 \mathrm{~b}$ ). Of the $7 \mathrm{~m}^{5} \mathrm{C}$ sites, 6 were highly methylated in all three biological replicates and the average wild type methylation levels ranged from 66 to $82 \%$. In contrast, one $\mathrm{m}^{5} \mathrm{C}$ site, $\mathrm{C} 960$ in mitochondrial SSU $18 \mathrm{~S}$ rRNA, was lowly methylated, with an average of $28 \%$ methylation (Fig. 3b). There were 6 rRNA species that were not methylated (Fig. 3a and Additional file 1: Table S3).

\section{NSUN5 is required for $\mathrm{m}^{5} \mathrm{C}$ at position C2268 in nuclear LSU 25S rRNA}

Two positions, C2268 and C2860, in nuclear LSU 25S rRNA were highly methylated in our RBS-seq datasets and both sites occur in the conserved domain IV of the large rRNA subunit in helices 70 and 89 , respectively. Recently, for the orthologous positions C2278 and C2870 in the yeast nuclear LSU 25S rRNA, the RMTases RCM1 and NOP2 were shown to be required for methylation, respectively $[13,15]$. Therefore, we predicted that the Arabidopsis homolog of RCM1, described here as NSUN5, and NOP2 paralogs described here as NOP2A/OLI2, $\mathrm{NOP} 2 \mathrm{~B}$ and NOP2C would be required for $\mathrm{m}^{5} \mathrm{C}$ at these sites $[25,42]$. To test these predictions we performed RBS-seq on nsun5, nop $2 a$, nop $2 b$ and nop $2 c$ mutants (Fig. 3c, Additional file 1: Figure S2B, S2C).

To test if NSUN5 is required for $\mathrm{m}^{5} \mathrm{C}$ at position C2268 of nuclear LSU $25 S$ rRNA we performed RBS-seq on total RNA from nsun5-1 and wild type (Fig. 3b). Methylation was reduced from $66 \%$ in wild type to $2 \%$ in nusn $5-1$ at position C2268 and methylation was not reduced at any other rRNA $\mathrm{m}^{5} \mathrm{C}$ sites. Similar results were obtained for a second, independent allele, nsun5-2 (Additional file 1: Figure S3D). Methylation of C2268 was reduced to $29 \%$ in nsun5-2. The low level of background methylation in nsun 5-2 may be due to low levels of NSUN5 expression in this mutant. While no transcripts were detected spanning the T-DNA insertion site, (Additional file 1: Figure S2C) spurious splicing may be occurring at low frequency to produce a small amount of functional, truncated protein. To confirm reduced methylation at position C2268 in nuclear 25S rRNA in nsun5 mutants, we developed a restriction enzyme digestion of PCR products using a dCAPs (derived cleaved amplified polymorphic sequences) primer derived from BS treated 25S rRNA. Cytosine methylation of $\mathrm{C} 2268$ retains the Hinfl restriction site and the enzyme cleaves the PCR products in wild type (Fig. $3 \mathrm{~d}$ and Additional file 1: Figure S3E). A reduction of C2268 methylation in nsun5-1 and nusn5-2 was observed by reduced cleavage of PCR products. Together these results demonstrate that C2268 25S rRNA is methylated by NSUN5 in Arabidopsis.

Next we tested if NOP2A, NOP2B or NOP2C were required for methylation at C2860 of nuclear LSU 25S rRNA by RBS-seq from the mutants (Fig. $3 \mathrm{~b}$ and Additional file 1 : Figure S3D). All mutants, nop $2 a$, nop $2 b$ and nop $2 c$ had wild type levels of methylation at C2860 25S rRNA, suggesting these RMTases do not methylate this site or are functionally redundant. To address this question, we attempted to identify nop $2 a$ nop $2 b$ double mutants, however these double mutants could not be identified from a segregating population. This suggests that NOP2A and NOP2B may act redundantly and are essential for plant viability. Sequence alignment of NOP2A, NOP2B and NOP2C revealed that NOP2B is missing motif IV, which is predicted to be involved in release of methylated RNA from the enzyme [54, 55] and NOP2C has an altered motif N1, which is involved in RNA binding, but is not essential for RMTase activity, as TRM4 homologs do not contain this motif [56] (Additional file 1: Figure S4B). Further research is required to uncover the RMTase(s) responsible for this $\mathrm{m}^{5} \mathrm{C}$ site and the redundancy of NOP2 paralogs in Arabidopsis. We 
A
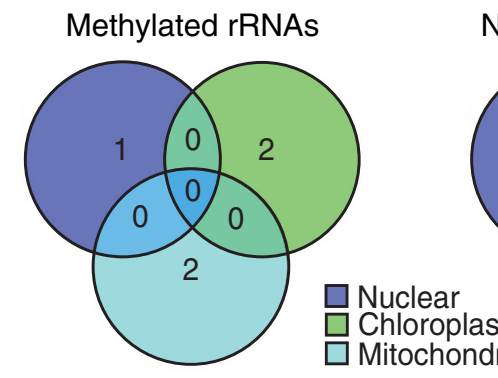

Non-methylated rRNAs

B

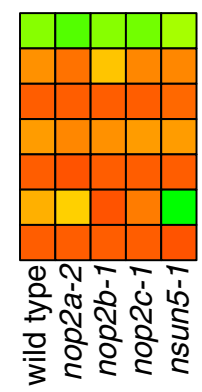

18S rRNA M C960

26S rRNA M C1586

16S rRNA C C916

23S rRNA C C1940

23S rRNA C C1977

25S rRNA N C2268

25S rRNA N C2860

C

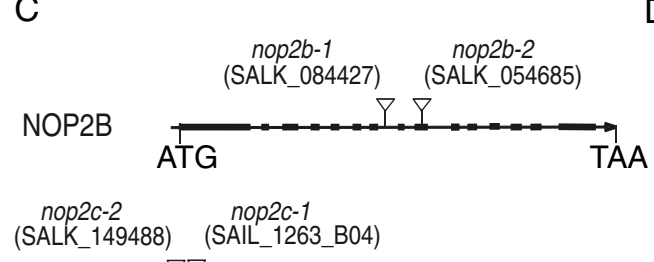

(SALK_149488) (SAlL_1263_B04)

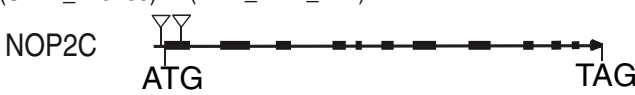

D
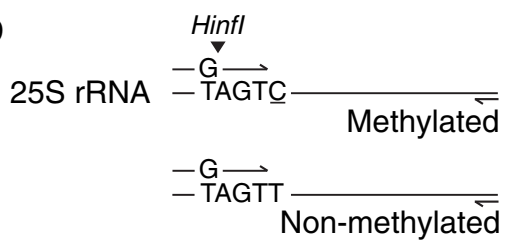

TAG

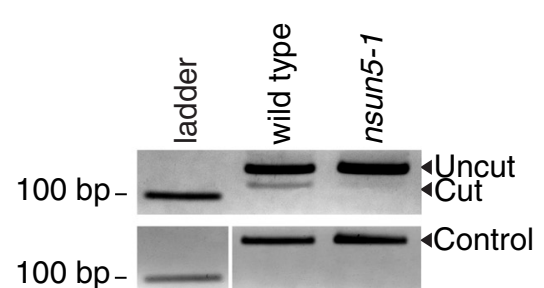

Fig. 3 NSUN5 methylates C2268 in Arabidopsis nuclear LSU 25S rRNA. a Genomic origins of methylated and non-methylated rRNA species. Methylated rRNAs were detected from all three genomes (3 biological replicates). $\mathbf{b}$ Left: Heatmap showing percentage methylation of cytosines in nuclear $(\mathrm{N})$, chloroplast (C) and mitochondrial (M) rRNA sequences in wild type and mutants nop2a-2, nsun5-1, nop2b-1 and nop2c-1. Cytosine positions are indicated next to rRNAs (3 biological replicates). Right: Partial secondary structure of 25S nuclear LSU rRNA helix 70 (domain IV) showing the cytosine position 2268 in red, which is methylated by NSUN5. c Genomic structure of nop2b, nop2c and nsun5 mutants showing T-DNA insertions (triangles) in exons (filled boxes). d Analysis of RNA methylation by NSUN5 at position C2268 on BS treated nuclear LSU 25S rRNA template. Above: Restriction maps of dCAPS amplified products showing the expected digest patterns of methylated and non-methylated template. The 25S_rRNA_F dCAPS primer contains a G mismatch at position four to generate a Hinfl restriction site when C2268 is methylated. Below: Cleavage of PCR amplified product by Hinfl confirms C2268 methylation in wild type as opposed to non-methylated C2268 in nsun5-1 results in loss of Hinfl restriction site. Loading control is undigested PCR product

also tested if the tRNA RMTases TRM4A, TRM4B and TRDMT1 methylate the remaining $6 \mathrm{~m}^{5} \mathrm{C}$ sites in rRNAs by RBS-seq from the mutants, trm $4 a$, trm $4 b-1$, trdmt1, trdmt1 trm $4 b$ and wild type (Additional file 1: Figure S3C). As expected, no reduction in rRNA methylation levels for the $7 \mathrm{~m}^{5} \mathrm{C}$ sites was observed in the mutants. Similarly, to demonstrate NOP2A and NSUN5 are rRNA specific and do not methylate tRNAs, we performed RBS-seq from both nop $2 a-2$ and nsun5-2. No reductions in $\mathrm{m}^{5} \mathrm{C}$ tRNA sites were observed (Additional file 1: Figure S3B).

tRNA and rRNA $\mathrm{m}^{5} \mathrm{C}$ sites are conserved from single-celled algae to multicellular plants

To test if methylated sites in nuclear tRNAs and organelle rRNAs are conserved through evolution, we constructed tRNA enriched RBS-seq libraries from six organisms; the single-celled algae, $N$. oculata, the multicellular 
macro algae C. taxifolia, and four vascular plants, the monocotyledonous plant $T$. durum, the dicotyledonous plants A. thaliana and B. rapa and the evolutionarily distinct ginkgophyte plant G. biloba. First, to identify transcribed tRNAs in non-Arabidopisis species, we mapped RNA-seq and RBS-seq to both our Arabidopsis tRNA isodecoder consensus sequences (Additional file 1: Table S1) and unique tRNA sequences from the closest relative with annotated tRNAs from the PlantRNA Database [49]. Similarly to construct species-specific rRNA references, we performed RNA-seq from total RNA from the five organisms and aligned the reads to either Arabidopsis rRNA references, species-specific rRNA references, or an Arabidopsis-rRNA guided assembled reference (Additional file 1: Table S3). These species-specific rRNA references were then utilized to align and annotate subsequent RBS-seq reads.
To test for conservation of $\mathrm{m}^{5} \mathrm{C}$ of tRNAs we performed RBS-seq on tRNA enriched libraries from $N$. oculata, $C$. taxifolia, T. durum, B. rapa, A. thaliana, and G. biloba and detected 35, 30, 51, 48, 56 and 34 tRNA isodecoders respectively (Fig. 4, Additional file 1: Table S2 and Table S4). Of these tRNAs, 30 were nuclear tRNAs, which are for the greater part methylated across all six species and the remaining 8 were putative chloroplast or mitochondrial tRNAs methylated in only one of the two species, $T$. durum or $N$. oculata. As these tRNAs were only methylated in one of the six species this may reflect chloroplast or mitochondrial tRNAs recently integrated into the nuclear genome of $T$. durum or $N$. oculata. Together these data demonstrate that methylation of chloroplast or mitochondrial encoded tRNAs is rare in the Kingdom Plantae and $\mathrm{m}^{5} \mathrm{C}$ methylation of tRNAs is generally restricted to nuclear-encoded tRNAs.

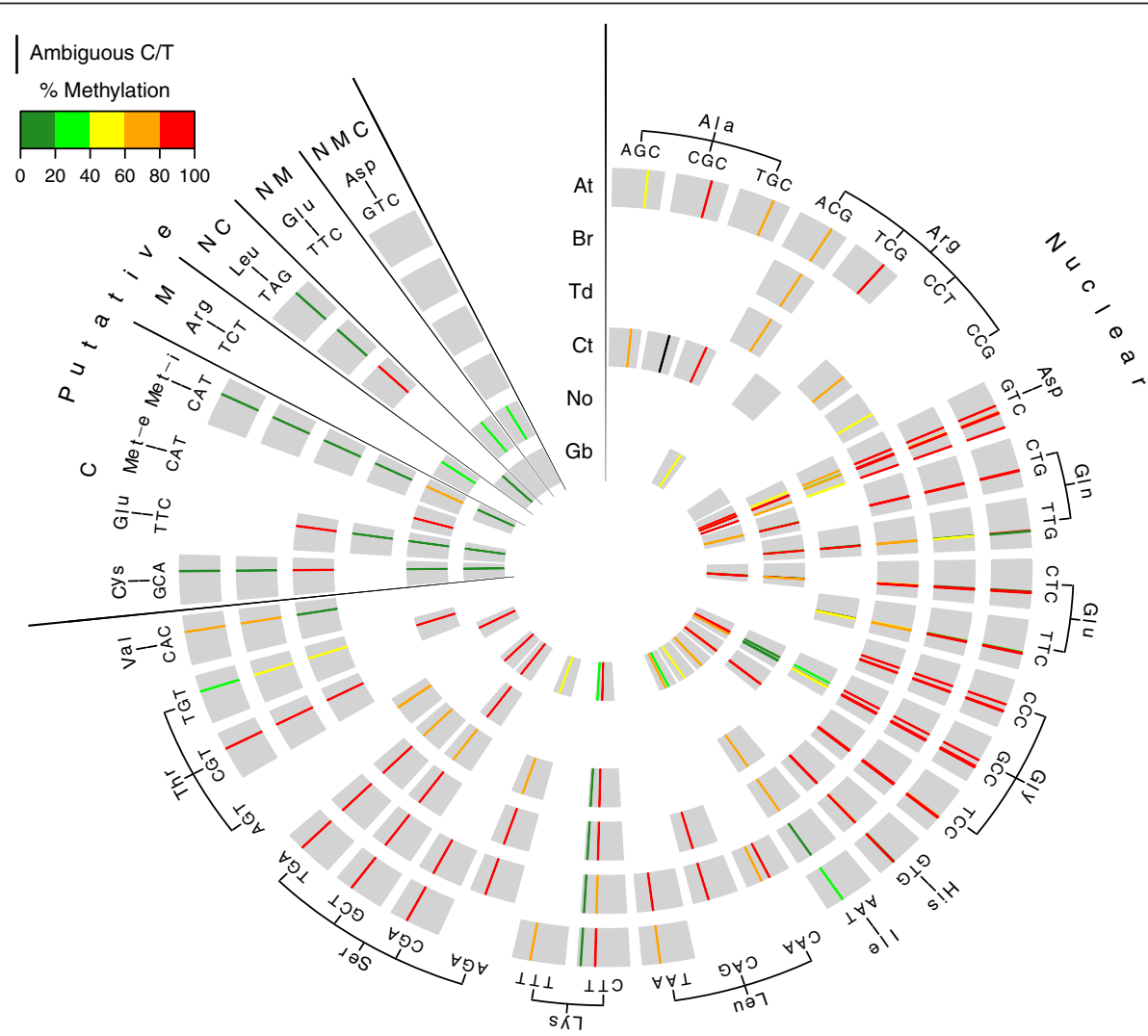

Fig. 4 Conservation of tRNA methylation in Kingdom Plantae. a Concentric circles from outer to inner represent Arabidopsis thaliana (At), Brassica rapa (Br), Triticum durum (Td), Nannochloropsis oculata (No), Caulerpa taxifolia (Ct) and Ginkgo biloba (Gb) tRNAs, respectively. The circles are split into two major sections for nuclear encoded tRNAs and tRNAs with putative genomic origins (nuclear- $\mathrm{N}$, chloroplast-C, mitochondrial-M). In each circle, individual tRNA consensus sequences are indicated as thick grey arcs and are organized alphabetically by amino acid, and then by anticodon. Specific tRNAs sequences for each species were aligned based on structural positions corresponding to the 72 bp representative tRNA structure. Cytosines that are methylated in at least one of the 6 species analysed are shown as a color-coded percentage methylation bar. The percentage methylation scheme used, Green = lowly methylated (0-40\%), red = highly methylated (80-100\%). Absence of a methylation bar indicates that the

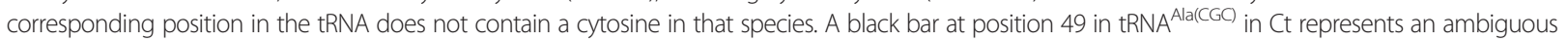
nucleotide which may be a C or T at this position. tRNAs that were not detected in the RBS-seq are not shown (Arabidopsis thaliana- 3 biological replicates and all other plant species 1 replicate) 
Detailed analysis of the 30 conserved nuclear tRNA isodecoders identified a total of 51 methylated positions. These 51 sites were divided into three classes, class one contained 35 highly conserved sites across all six species, class two contained 5 highly conserved sites in five species and the other species contained a single-nucleotide polymorphism (SNP) and the third class contained 11 sites which are methylated in at least one species and not methylated in the other species. Class two that contained SNPs, were either transitions $(C>T)$ or transversions $(C>G)$ at the methylated positions. An example of a transversion occurs in tRNA ${ }^{\text {Asp(GTC) }}$. At position C50 in tRNA ${ }^{\mathrm{Asp}(\mathrm{GTC})}$ in C. taxifolia had a transversion from $\mathrm{C}$ to $\mathrm{G}$, abolishing an otherwise highly conserved $\mathrm{m}^{5} \mathrm{C}$ site. The $\mathrm{G}$ transversion was confirmed by using RNAseq. An example of class three, $\mathrm{m}^{5} \mathrm{C}$ site reduction in one species, was position C48 of tRNA ${ }^{\mathrm{Glu}(\mathrm{CTC})}$. While T.durum and G.biloba had low levels of methylation (22-40\%) at C48, three other species were not methylated at this site, despite the presence of a cytosine residue in non-BS converted RNA.

Within class three, containing conserved cytosine residues methylated in at least one species, a noteworthy example was tRNA ${ }^{\mathrm{Gln}(\mathrm{TTG})}$ which contained methylated positions in all species however sites were not conserved. For example, in T. durum and N. oculata positions C48 and $\mathrm{C} 49$ were both methylated however in the other tested species only C48 or C49 was methylated, but not both sites despite the presence of cytosines at these positions. This site variability was also identified by Blanco et al. [18], as mice are methylated at one site in tRNA ${ }^{\mathrm{Gln}(\mathrm{TTG})}$, while humans are methylated at two sites. A clearer understanding of the other ribonucleotide modifications near these tRNA positions may provide further insight into these observations.

We also identified two additional $\mathrm{m}^{5} \mathrm{C}$ structural positions, C34 and C68 in tRNA ${ }^{\mathrm{Leu}(\mathrm{CAA})}$ and tRNA ${ }^{\mathrm{Lys}(\mathrm{CTT})}$ of $B$. rapa and G. biloba, respectively, that were not methylated in other species. tRNA ${ }^{\mathrm{Leu}(\mathrm{CAA})}$ position C34 methylation was only detected in B. rapa and G. biloba at 89 and $20 \%$, respectively. The variation of methylation at this position may be due to environmental factors, as methylation at this site in yeast was previously shown to be altered under oxidative stress conditions [10]. It is predicted that tRNA ${ }^{\mathrm{Leu}(\mathrm{CAA})}$ position C34 is methylated in Arabidopsis but we did not detect Arabidopsis tRNA ${ }^{\mathrm{Leu}(\mathrm{CAA})}$ in our datasets. For tRNA ${ }^{\text {Lys(CTT) }}$ position C68, G. biloba had $25 \%$ methylation while $A$. thaliana, B. rapa and $T$. durum had very low methylation (below our $20 \%$ methylation threshold). Similarly, methylation at nearby structural positions C67 and C69 in other tRNAs has also been reported in humans [30].

Conservation of rRNAs $\mathrm{m}^{5} \mathrm{C}$ sites was tested amongst all six organisms, $N$. oculata, C. taxifolia, T. durum, B. rapa,
A. thaliana, and G. biloba, by RBS-seq from total RNA. A total of 8 highly conserved $\mathrm{m}^{5} \mathrm{C}$ sites in nuclear, chloroplast and mitochondrial structural positions of LSU and SSU rRNAs were identified (Fig. 5 and Additional file 1: Table S3 and Table S5). Interestingly, methylation of LSU 25S rRNA cytosines C2268 and C2860, which are predicted to be dependent upon homologs of NSUN5 and NOP2A/ $\mathrm{NOP} 2 \mathrm{~B} / \mathrm{NOP} 2 \mathrm{C}$, respectively are conserved in all six species $[13,15]$. Six of these $8 \mathrm{~m}^{5} \mathrm{C}$ sites were highly conserved in methylation percentage and position across all tested species except C916 in SSU 16S chloroplast rRNA for which the methylation across species ranged from 31 to $87 \%$. The remaining two highly conserved sites, mitochondrial C960 in SSU 18S rRNA and C1549 in LSU 26S rRNA were highly methylated in four of the six tested species. A further eight $\mathrm{m}^{5} \mathrm{C}$ sites, were species specific of which 6, C1703 and C1713-1717, occurred in a 15 bp region on $T$. durum nuclear SSU $18 \mathrm{~S}$ rRNA and the other two methylated sites, C1566 mitochondrial SSU $18 S$ rRNA and C1887 chloroplast LSU 23S rRNA occurred only in $N$. oculata. The five clustered $\mathrm{m}^{5} \mathrm{C}$ sites in $18 \mathrm{~S}$ rRNA maybe attributed to BS non-conversion events as a result of strong secondary structure of the rRNA. The remaining species-specific sites in $N$. oculata may reflect species-specific factors regulating translation by ribosomes.

\section{Discussion}

Here, we show that the post-transcriptional modification 5 -methylcytosine is only detected on nuclear-encoded tRNAs of plants however methylation of rRNAs occurs in transcripts from all three organelles. Strong conservation of tRNA and rRNA methylated sites were observed in species ranging from single-celled algae to multicellular plants. Furthermore, in Arabidopsis thaliana, the evolutionarily conserved RNA methyltransferases TRM4B and TRDMT1 were found to be required for tRNA methylation at multiple nucleotide sites, while NSUN5 specifically methylates nuclear LSU 25S rRNA at position C2268.

Our study detected 39 candidate sites for 5-methylcytosine in Arabidopsis nuclear tRNAs and an additional 20 $\mathrm{m}^{5} \mathrm{C}$ sites were detected across diverse plant species and all sites except one are new discoveries in plants. The majority of $\mathrm{m}^{5} \mathrm{C}$ sites were found at positions within tRNA secondary structure known to have 5methylcytosine in animals [2, 3, 18, 29-31], broadly supporting existing expectations of the role of $\mathrm{m}^{5} \mathrm{C}$ in modulating tRNA function [2]. An emerging facet of tRNA biology in both plants and animals is their processing into smaller regulatory RNAs [57-61], and TRDMT1- mediated addition of $\mathrm{m}^{5} \mathrm{C}$ has been demonstrated to protect tRNAs against heat and oxidative stress-mediated cleavage in Drosophila [12]. Likewise, methylation by TRM4/NSUN2 in humans and mouse has been demonstrated to protect tRNAs from oxidative stress 


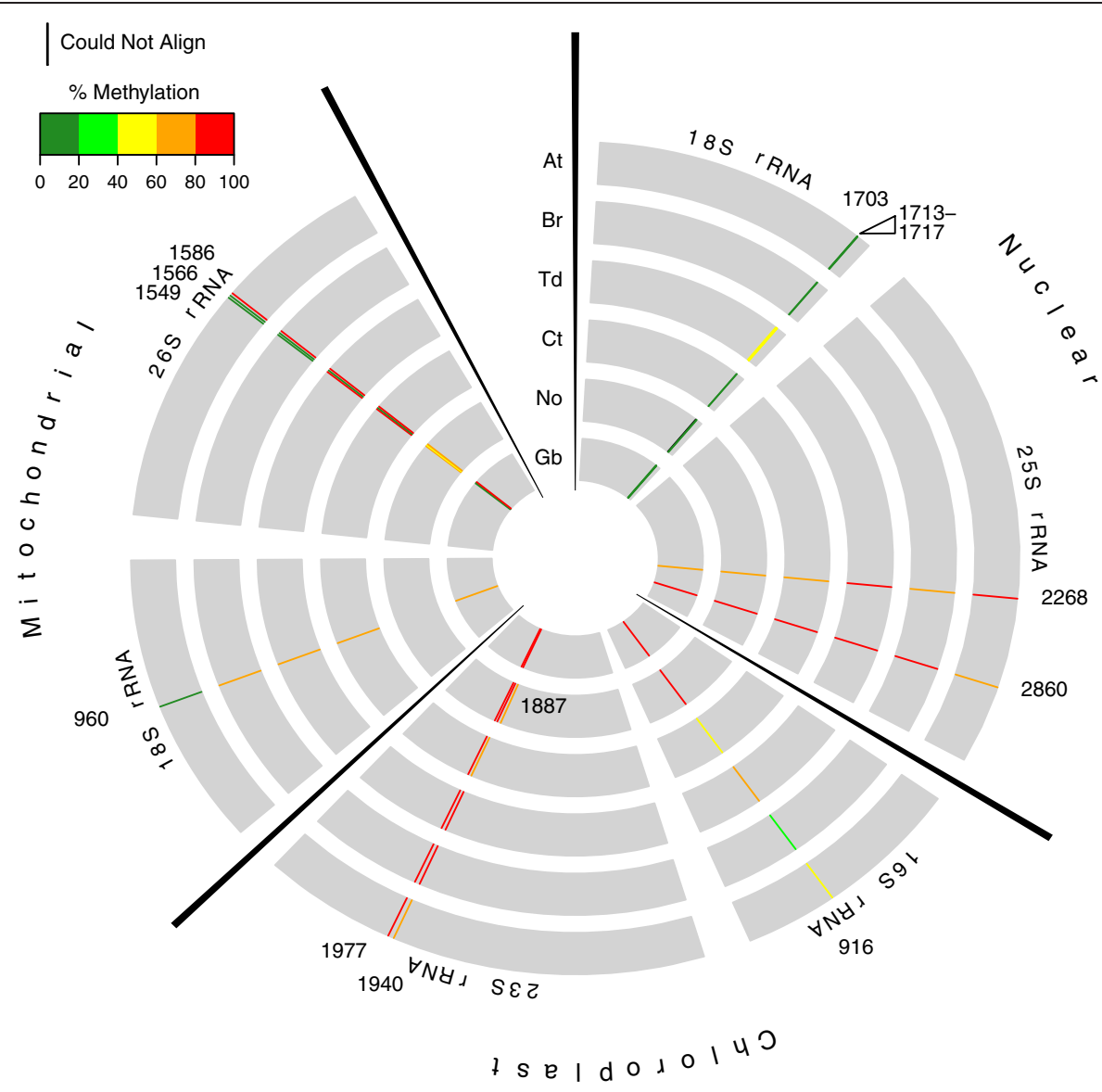

Fig. 5 Conservation of rRNA methylation in Kingdom Plantae. a Concentric circles from outer to inner represent Arabidopsis thaliana (At), Brassica rapa (Br), Triticum durum (Td), Nannochloropsis oculata (No), Caulerpa taxifolia (Ct) and Ginkgo biloba (Gb) rRNAs, respectively. The circles are split into three sections for nuclear, chloroplast and mitochondrial encoded rRNAs. In each circle, individual rRNA sequences are represented as thick grey bars. The nucleotide positions for each rRNA species are based on alignment with the corresponding Arabidopsis consensus sequences. Cytosines that are methylated in at least one of the 6 species analysed are shown as a color-coded percentage methylation bar. In the percentage methylation scheme used, Green = lowly methylated (0-40\%), red = highly methylated (80-100\%). Absence of a methylation bar indicates that the corresponding position in the rRNA does not contain a cytosine in that species. Open triangle indicates where consecutive cytosines are methylated in Triticum durum. The black methylation bar at position 1703 in Nannochloropsis oculata SSU 18 S rRNA shows where the sequence could not be aligned to the Arabidopsis reference sequence (At 3 replicates and other species 1 replicate)

induced cleavage [18]. Together, this data provides a wealth of $\mathrm{m}^{5} \mathrm{C}$ sites mediated by TRDMT1 and TRM4B that can now be interrogated for a role of this phenomenon in plants. Future experiments will determine if increased cleaved tRNA fragments are observed in the RMTase mutants and testing these mutants under various environmental conditions may highlight additional roles for these genes in modulating stress responses.

Detection of $\mathrm{m}^{5} \mathrm{C}$ sites on only nuclear tRNAs in Arabidopsis is consistent with the mitochondrial and chloroplast genomes being derived from alpha-proteobacteria [62] and cyanobacterial ancestors [63], respectively. Complementary to our data, $\mathrm{m}^{5} \mathrm{C}$ sites were not detected on tRNAs from bacterium Escherichia coli and Bacillus subtilis [3]. In contrast, six mitochondrial tRNAs in bovine [32] and five mitochondrial tRNAs in human [29, 64] contain methylation at positions $\mathrm{C} 48, \mathrm{C} 49$ and $\mathrm{C} 72$. These data suggest that methylation of mitochondrial tRNAs may have evolved independently in animals since the last common ancestor between plants and animals. A lack of methylation of mitochondrial and chloroplast tRNAs was generally conserved across diverse plant species. Three notable exceptions were chloroplast-like tRNA ${ }^{\mathrm{Cys}(\mathrm{GCA})}$, tRNA $^{\text {Glu(TTC) }}$ and tRNA ${ }^{\text {Leu(TAG) }}$ in $T$. durum that we detected methylation however no methylation was observed in the homologues of other plants species. One interpretation of these observations is that the three chloroplast-like tRNAs of $T$. durum represent recent DNA integration events into the nucleus. After nuclear integration and transcription, these tRNAs are methylated by RMTases, presumably TRM4B, TRDMT1 or RCMT9. 
Many mitochondrial genes and tRNAs are often incorporated into nuclear genomes and accordingly lost from the organelle genomes over time [46]. As a result, to obtain the full complement of tRNAs required for translation of mitochondrial encoded proteins, requires the import of tRNAs from the nucleus. Nine tRNA isoacceptors are predicted to be imported from the nucleus to the mitochondrion in Arabidopsis [44] and several of these tRNAs, such as tRNA ${ }^{\text {Gly(CCC) }}$ were found to be methylated in our data. We speculate that $\mathrm{m}^{5} \mathrm{C}$ methylation by TRM4B and/or TRDMT1 of these mitochondrial-imported nuclear tRNAs occurs in the cytoplasm before import into the mitochondria. Methylation of nuclear, eukaryotic tRNAs that are imported into the mitochondria implies they are not inherently incompatible with the prokaryotic mitochondrial translation machinery.

Nine putative cytosine RMTase enzymes including TRDMT1/DNMT2 are encoded in the Arabidopsis genome of which we demonstrate TRM4B and TRDMT1 methylate tRNAs and not rRNAs [25, 26]. Duplication of TRM4 resulted in two paralogs, TRM4A and TRM4B in Arabidopsis. TRM4B retains methyltransferase activity on tRNAs while TRM4A contains a deletion of motif I which is required for target cytosine binding [52]. We cannot rule out that TRM4A contains other regulatory functions, for example regulating $\mathrm{m}^{5} \mathrm{C}$ stability or modulating accessibility of $\mathrm{m}^{5} \mathrm{C}$ sites to RNA binding proteins. TRM4B methylation of tRNAs at positions C48, C49 and C50 is consistent with the fact that animal and yeast homologues also methylate these tRNA structural positions [2, 3, 16, 18, 29-31]. NSUN2/TRM4 in human has been found to methylate tRNA(s) at position C72 [30]. In contrast, we identified only

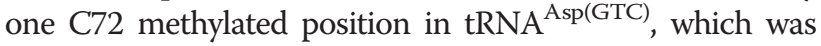
independent of TRM4B. TRDMT1 has been previously described as a tRNA C38 specific RMTase, in animals and methylates tRNA ${ }^{\text {Asp(GTC), }}$ tRNA $^{\text {Gly(GCC) }}$ and tRNA ${ }^{\operatorname{Val}(\mathrm{AAC})}$ $[11,12,30]$. We confirmed this C38 specific observation in Arabidopsis by not only detecting the previously described tRNA $^{\text {Asp(GTC) }}$, but also two new tRNAs, tRNA ${ }^{\mathrm{Gly}(\mathrm{GCC})}$ and

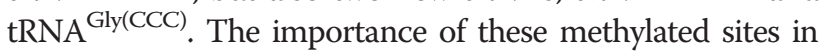
tRNAs is illustrated in other organisms, as loss of TRM4 and TRDMT1 results in reduced abundance of mature tRNAs and translational efficiency in mice [11]. In addition, tRNA $\mathrm{m}^{5} \mathrm{C}$ sites mediated by TRM4 in yeast are required for tolerance to the antibiotic paromomycin, which is an aminoglycoside, like hygromycin B [65]. Similarly our data of trm $4 b$ mutants increased sensitivity to hygromycin B when compared to wild type suggests TRM4B methylated tRNAs have a role in translational efficiency. Interestingly the loss of both TRDMT1 and TRM4B resulted in a severe sensitivity to hygromycin B. There are only three tRNAs which we found to be methylated by both TRDMT1 and TRM4B suggesting that the $\mathrm{m}^{5} \mathrm{C}$ mediated structure of these three tRNAs is important for translation. Translation is tightly regulated in order for organisms to adapt quickly to environmental stresses, such as oxidative and heat stress [10, 66]. Alterations in translation in Arabidopsis trdmt1 trm $4 b$ mutants may affect translational regulation under stress conditions and reduce plant fitness.

Our identification of $\mathrm{m}^{5} \mathrm{C}$ sites at tRNA structural positions C48, C49, C50 and C72 independent of either TRM4B or TRDMT1 is similar to recent observations in mouse using RBS-seq and raises the possibility that another RMTase methylates these sites [18]. This is in contrast to humans where all tRNA $\mathrm{m}^{5} \mathrm{C}$ sites are dependent on either TRM4 or TRDMT1 [18]. We propose that the additional tRNA RMTase in plants is the closest TRM4 homologue RCMT9 (At5g66180). This hypothesis could be tested by identification of $r \mathrm{cmt} 9$ mutants and performing RBS-seq on enriched tRNAs as described here. An alternative hypothesis is that the TRM4 and TRDMT1 independent $\mathrm{m}^{5} \mathrm{C}$ sites are not $\mathrm{m}^{5} \mathrm{C}$ sites but other cytosine modifications that are resistant to BS conversion [29, 51]. This could be tested by performing mass spectrometry on purified tRNAs from plant trm $4 b$ trdmt1 double mutants and determining the presence or absence of $\mathrm{m}^{5} \mathrm{C}$. In nsun2 trdmt1 double mutant mice, RNA $\mathrm{m}^{5} \mathrm{C}$ levels were reduced by at least $90 \%$ compared to wild type mice [11]. It is unclear whether this indicates that additional $\mathrm{m}^{5} \mathrm{C}$ sites in tRNAs remain, or if the detected $\mathrm{m}^{5} \mathrm{C}$ was derived from contaminating rRNA. We favour the first hypothesis that these TRM4B and TRDMT1 independent sites are bona fide $\mathrm{m}^{5} \mathrm{C}$ sites as they are in tRNA structural positions which normally contain $\mathrm{m}^{5} \mathrm{C}$ and that these sites are methylated by Arabidopsis RCMT9, which shares sequence homology with TRM4 homologues [25].

Our approach not only detected methylated sites in RNAs but also provides a quantitative measure of the percentage of transcripts having this modification. This allowed us to undertake a quantitative comparative analysis of methylation in more than 200 individual sites in tRNAs and 50 individual sites in rRNAs amongst diverse plants. Interestingly both the percent methylation and specific sites in tRNAs and rRNAs were broadly conserved across the six plant species. This strong conservation strongly supports the functional importance for these $\mathrm{m}^{5} \mathrm{C}$ sites in roles such as regulating the structure and stability of rRNAs and tRNAs [2].

Our study detected 7 candidate sites for 5-methylcytosine in Arabidopsis nuclear, chloroplast and mitochondrial LSU and SSU rRNAs, all of them novel in plants. Many of these high-confidence sites were found at positions within rRNA regions known to have 5-methylcytosine in animals and bacteria [2, 12, 14, 33-36]. Of note, we did not detect LSU 5S rRNA methylation in any plant species analyzed, while in contrast, this rRNA species is methylated by TRM4/ NSUN2 in HeLa cells [30, 31]. It is intriguing that while plant chloroplast and mitochondrial tRNAs are devoid of 
$\mathrm{m}^{5} \mathrm{C}$, organelle rRNAs contain $\mathrm{m}^{5} \mathrm{C}$. It is unknown whether the rRNAs are methylated inside the chloroplast and mitochondria, or if they are exported to allow addition of modifications from nuclear derived modifying enzymes before they are imported back into the organelles. Arabidopsis RMTases NOP2B and RNMT/FMU are both predicted to localize in chloroplasts [26]. This suggests that these RMTases methylate the rRNA inside the organelles. Further study is required on the location of the RMTases, to confirm these findings and to assess where catalysis of $\mathrm{m}^{5} \mathrm{C}$ occurs.

There are five RMTases present in the Arabidopsis genome, which are predicted to methylate rRNA, based on sequence similarity to rRNA RMTases in other organisms. In this study, we investigated the rRNA $\mathrm{m}^{5} \mathrm{C}$ sites requiring the RCM1 homolog, NSUN5 and the NOP2 paralogs NOP2A, NOP2B and NOP2C. Here we showed that Arabidopsis NSUN5 is required for methylation of C2278 in nuclear LSU 25S rRNA. Interestingly, the yeast NOP2 ortholog in Arabidopsis, NOP2A was not found to be required for $\mathrm{m}^{5} \mathrm{C}$ at C2860 of nuclear LSU 25S rRNA. We hypothesise that this may be due to functional redundancy with the other NOP2 paralogs in the Arabidopsis genome, NOP2B and NOP2C. It is uncertain if the paralogs NOP2B and NOP2C are functional RMTases. NOP2B lacks motif IV, which is involved in release of target RNA after methylation by motif VI $[54,55]$. In yeast, a point mutation in motif IV of the conserved residue Cys ${ }^{424}$ in NOP2 leads to accumulation of mutant nop 2 protein-RNA complexes and cell death $[54,67]$. It is possible that NOP2B is utilizing a Cys residue contained in a highly diverged, nonconforming motif IV to evade cell death. An alternative possibility is that although the $\mathrm{m}^{5} \mathrm{C}$ site is conserved, that another RMTase in the genome is responsible for methylation at this site. The most promising candidate for this possibility is the Arabidopsis homolog of bacterial Fmu, RNMT, which is predicted to methylate rRNA [25, 43].

Conservation of the enzymes and methylation sites in rRNA across species suggests conservation of function. The possible functions include, regulation of protein synthesis, stability and maturation of rRNA and translational fidelity. It remains to be seen if the phenotype of nop $2 a$ can be linked to any specific $\mathrm{m}^{5} \mathrm{C}$ sites, or alterations in rRNA processing. The rRNA $\mathrm{m}^{5} \mathrm{C}$ sites and the mutants identified in this work provide a platform to launch studies into the roles of specific rRNA $\mathrm{m}^{5} \mathrm{C}$ sites under different environmental conditions.

\section{Conclusions}

This comprehensive characterization of the tRNA and rRNA methylation profiles in plants uncovered nuclear specific methylation of tRNAs, while rRNAs are methylated from all three genomes. The method of enriching for tRNAs combined with RBS-seq on wild type and mutants allowed us to identify $\mathrm{m}^{5} \mathrm{C}$ sites dependent on NSUN2/TRM4 and to extend the known target range of TRDMT1 to an additional two tRNAs at position C38 in plants. We also determined that NSUN5 is required for $\mathrm{m}^{5} \mathrm{C}$ at $\mathrm{C} 2278$ in nuclear LSU 25S rRNA and uncovered functional redundancy among the Arabidopsis NOP2 paralogs, NOP2A, NOP2B and NOP2C, as loss of one of these three enzymes is insufficient to remove any rRNA $\mathrm{m}^{5} \mathrm{C}$ sites, while loss of both NOP2A and NOP2B appears lethal. Arabidopsis RMTase enzymes are encoded in the nuclear genome. This suggests movement of either the nuclear encoded RMTase enzymes to organelles, or transport and re-import of organelle rRNAs. We favour the former hypothesis as the Arabidopsis Fmu-like RNMT and NOP2B are predicted to be located in organelles [26], which suggests that they methylate rRNA inside the mitochondria and chloroplast, and that NOP2B acts redundantly with NOP2A and NOP2C. While several mitochondrial tRNAs are methylated in vertebrates [29, 32, 64], our data suggests that like bacterial tRNAs, plant mitochondrial and chloroplast tRNAs are not methylated [3]. This suggests that vertebrates gained the ability to methylate mitochondrial tRNAs during evolution. We discovered high levels of conservation of tRNA and rRNA methylation across diverse plant species, including the sites shown to be methylated by TRDMT1, TRM4B and NSUN5 in Arabidopsis, indicating that these enzymes are most likely responsible for methylation at these sites in other Plantae. The conservation of RMTases and $\mathrm{m}^{5} \mathrm{C}$ sites strongly suggests important, conserved functions, which deserve investigation. We investigated the function of tRNA $\mathrm{m}^{5} \mathrm{C}$ sites using the antibiotic hygromycin B. Loss of both TRDMT1 and TRM4B resulted in increased sensitivity of mutants to the antibiotic hygromycin B, suggesting that tRNA $\mathrm{m}^{5} \mathrm{C}$ sites affect tRNA structure in a combinatorial manner. Our data provides the foundation and characterization of Arabidopsis genetic mutants needed to further probe the functions of RNA $\mathrm{m}^{5} \mathrm{C}$ in plants.

\section{Methods}

\section{Plant material and growth conditions}

A. thaliana (Columbia ecotype) and B.rapa plants were grown in Phoenix Biosystem controlled environment rooms at $21^{\circ} \mathrm{C}$ under metal halide lights that provided a level of PAR (photosynthetic active radiation) of 110 $\mu \mathrm{mol}$ of photos $/ \mathrm{m}^{2} / \mathrm{s}$. Plants were grown under long day photoperiod conditions of $16 \mathrm{~h}$ light and $8 \mathrm{~h}$ darkness on soil (Debco Seedling raising mix) or $1 / 2$ MS media supplemented with $1 \%$ sucrose. G. biloba was grown at the Botanic Gardens of Adelaide (34.9181 ${ }^{\circ}$ S, $138.6107^{\circ}$ E, Australia). C. taxifolia was grown in artificial seawater at $25^{\circ} \mathrm{C}$ under fluorescent lights that provided PAR of 80 
$\mu$ mol of photos $/ \mathrm{m}^{2} / \mathrm{s}$. N. oculata was grown in artificial seawater supplemented with nitrogen and phosphorous under fluorescent lights that provided PAR of $40 \mu \mathrm{mol}$ of photos $/ \mathrm{m}^{2} / \mathrm{s}$ to $\log$ phase. For Arabidopsis hygromycin $\mathrm{B}$ assays, seeds were sown on control and hygromycin B [15 ug/mL] supplemented $1 / 2$ MS media with $1 \%$ sucrose. Hygromycin B was purchased from A.G. Scientific, Inc.. Plants were photographed with a Canon PowerShot G15 camera at 10 and 20 days after germination (DAG).

Mutant alleles described are; nop2a-2 (oli2-2) (SALK_ 129648), nop $2 b-1$ (SALK_084427), nop $2 b-2$ (SALK_0546 85), nop2c-1 (SAIL_1263_B04), nop2c-2 (SALK_149488), nsun5-1 (SALK_204104), nsun5-2 (SALK_004377), trdmt1 (SALK_136635), trm4a (SALK_121111), trm4b-1 (SAIL 318_G04) and trm4b-2 (SAIL_667_D03). The trdmt1 trm4 $\bar{b}$ double mutants were generated using the trdmt1 and the trm $4 b-1$ mutant alleles. The nop $2 a$ nop $2 b$ double mutants were generated using the nop $2 a-2$ (oli2-2) and the nop $2 b-1$ mutant alleles. Primers used to identify homozygous T-DNA mutants are provided (Additional file 1: Table S6).

Nucleotide sequence data for the following genes are available from The Arabidopsis Information Resource (TAIR) database under the following accession numbers: NOP2A/OLI2 (At5g55920), NOP2B (At4g26600), NOP2C (At1g06560), NSUN5 (At5g26180), TRDMT1 (At5g25480), TRM4A (At4g40000), TRM4B (At2g22400).

\section{RNA isolation and bisulfite conversion of RNA}

Total RNA was isolated from either 10 day old $A$. thaliana seedlings, or $A$. thaliana floral buds, $T$. durum flag leaf, $B$. rapa and G. biloba shoot apexes, C. taxifolia fronds or log phase growth $N$. oculata cells using the Spectrum Plant total RNA kit (SIGMA-ALDRICH) and contaminating DNA removed using DNase (SIGMA-ALDRICH). To enrich for tRNAs, $10 \mu \mathrm{g}$ of total RNA was separated on a $10 \%$ polyacrylamide gel, the region containing 65-95 nts was removed and RNA was purified. Either total RNA or purified tRNAs were used for library construction using Illumina's TruSeq RNA sample kit v2, as per the manufacturer's instructions. As bisulfite treated RNA is sheared, bisulfite treated samples were quickly processed after addition of the fragmentation buffer. For bisulfite conversion, $200 \mathrm{pg}$ of control in vitro transcribed Renilla luciferase (R-Luc) RNA was added to either $2 \mu \mathrm{g}$ of total RNA or $200 \mathrm{ng}$ of purified tRNAs and converted with sodium metabisulfite (SIGMA-ALDRICH) as previously described $[29,51]$. Bisulfite treated total RNA or purified tRNAs were used as template for Illumina library construction as described above. Illumina sequencing was performed on a MiSeq platform at ACRF, Adelaide. For a description of the sequenced libraries, see (Additional file 1: Table S7).

\section{Sequence read mapping and methylation analysis}

Sequences were trimmed for adapters and filtered for low quality reads using CLC Genomics Workbench (Qiagen). In order to identify tRNAs and to reduce mapping ambiguity, we collapsed identical and highly similar tRNA isodecoder sequences from The Arabidopsis Information Resource [68] to create a reference consensus list of 100 tRNA isodecoders (Additional file 1: Table S1). For tRNAs from other species, both the Arabidopsis reference consensus list and unique tRNA sequences obtained from the closest relative available in the PlantRNA Database [49], were used. rRNA sequences were obtained from the NCBI Refseq database where available. For species without available rRNA sequences, RNA-seq reads were aligned to the Arabidopsis RNA reference sequence for the corresponding rRNA subunit and a consensus was derived in order to obtain species-specific SNPs (Additional file 1: Table S3). RBS-seq reads of tRNAs and rRNAs were mapped to in silico converted reference sequences, while RNA-seq reads were mapped to unconverted sequences. CLC Genomics Workbench (Qiagen) was used to align sequence reads to the corresponding tRNA and rRNA reference sequences. In order to compare structural positions in tRNAs with different sequences and lengths, the representative structure model was used [69]. For rRNAs, the reference sequences of all plant species analysed were aligned to the Arabidopsis references and the numbering was based on the nucleotide position of the corresponding Arabidopsis rRNA reference sequence.

In order to identify methylated cytosines, non-conversion of a cytosine in read sequences was taken to indicate the presence of $\mathrm{m}^{5} \mathrm{C}$. Renilla Luciferase in vitro transcribed mRNA lacking $\mathrm{m}^{5} \mathrm{C}$ was used to ensure that conversion efficiency was greater than $98 \%$. To ensure robust detection of methylated sites in tRNAs and rRNAs, a minimum of 5 reads coverage was required and a minimum methylation level of $20 \%$ ( $\leq 80 \%$ conversion rate). Percentage methylation at specific positions was calculated as the number of mapped cytosines divided by the combined total number of mapped cytosines and mapped thymines. Heatmaps showing percentage methylation were created in $\mathrm{R}$ [70], using the R package "Pretty heatmaps" [71].

\section{Methyl-chop PCR methylation assay}

cDNA derived from the bisulfite treated RNA was used to generate PCR products from tRNA Asp(GTC) and helix 70 of $25 \mathrm{~S}$ nuclear rRNA using the primers forward Asp tRNA_At_FWD and reverse Asp tRNA_At_REV and primers forward 25S_rRNA_F and reverse 25S_rRNA_R, respectively. The 25S_rRNA_F dCAPS primer contains a G mismatch at position four from the 3' end to generate a HinfI restriction site. PCR products were digested with HpyCH4IV or HinfI restriction enzymes (New England Biolabs), respectively. The tRNA ${ }^{\text {Asp(GTC) }} 72 \mathrm{bp}$ PCR product 
is digested by $H p y C H 4 I V$ resulting in two digestion products of 35bp and 37bp if C38 is methylated, and is undigested if $\mathrm{C} 38$ is non-methylated and thus converted to T38 by bisulfite treatment, causing the HpyCH4IV restriction enzyme site to be lost. The $25 \mathrm{~S}$ rRNA PCR product is 155bp in length. When C2268 is methylated, the restriction enzyme Hinfl cleaves the PCR product into two fragments of 29bp and 126bp. Non-methylated $25 \mathrm{~S}$ rRNA has a T at position 2268 after bisulfite treatment, and this eliminates the Hinfl restriction enzyme site, leaving the product at $155 \mathrm{bp}$ and undigested. Undigested PCR products were used as loading controls. Primer sequences used are provided (Additional file 1: Table S6).

\section{Semi-quantitative PCR}

Semi-quantitative PCR was performed using an Invitrogen SuperScript III kit as per the manufacturer's recommendations from $2 \mu \mathrm{g}$ of total RNA and oligo-dT primed cDNA synthesis. Semi-quantitative PCR detection of TRM4A, TRM4B, NSUN5, NOP2B and NOP2C mRNA was performed using the primers provided (Additional file 1: Table S6). For the RNA input control, amplification of the housekeeping gene PDF2A was used with primers forward PDF2_RT-PCR_F and reverse PDF2_RT-PCR_R. Quantitative PCR was performed to test NOP2C mRNA abundance using Roche LightCycler480 and SYBER green.

\section{Availability of supporting data}

The data sets supporting the results of this article are available in NCBI's GEO database repository, and are accessible through GEO Series accession numbers GSE68444, GSE68445, GSE68447 and GSE68448.

\section{Additional file}

Additional file 1: Figure S1. Efficient bisulfite conversion of nonmethylated cytosine residues. Figure S2. Characterization of Arabidopsis thaliana T-DNA mutants. Figure S3. Specificity of tRNA and rRNA MTases. Figure S4. Multiple sequence alignment of methyltransferase motifs from Arabidopsis thaliana RMTases. The amino acid sequences of two subfamilies of RMTases in Arabidopsis; (A) TRM4A and TRM4B; (B) NOP2ANOLI2, NOP2B and NOP2C were aligned using Clustal Omega [72]. Table S1. Unique tRNA isodecoder consensus sequences used for tRNA expression and methylation analysis. Table S2. Annotation table of tRNA isodecoder sequences detected in diverse plant species. Table S3. Ribosomal RNA sequences used for methylation analysis in diverse plant species and number of $\mathrm{m}^{5} \mathrm{C}$ sites Table S4. Methylation \% of tRNAs from A. thaliana, B. rapa, T. durum, C. taxifolia, N. occulata and G. biloba. Table S5. Methylation \% of rRNAs from A. thaliana, B. rapa, T. durum, C. taxifolia, N. occulata and G. biloba. Table S6. Primer sequences used in this study [73]. Table S7. Read coverage of libraries sequenced. (ZIP $5681 \mathrm{~kb}$ )

\section{Abbreviations}

rRNA: Ribosomal RNA; tRNA: Transfer RNA; $m^{5} \mathrm{C}$ : 5-methylcytosine; RMTase: RNA methyltransferase; TRM4: Transfer RNA methyltransferase 4; TRDMT1: Transfer RNA Asp methyltransferase 1; RNA-seq: Illumina RNA sequencing; RBS-seq: Illumina RNA BiSulfite sequencing; NOP2: Nucleolar Protein 2; OLI2: Oligocellula 2; NSUN2: NOP2/Sun domain protein 2; NSUN5: NOP2/Sun domain protein 5; BS: Bisulfite; R-Luc: Renilla luciferase;
RCMT9: RNA cytosine methyltransferase 9; AdoMet: S-adenosyl-L-methionine; dCAPS: Derived cleaved amplified polymorphic sequences;

PAR: Photosynthetic active radiation.

\section{Competing interests}

The authors declare that they have no competing interests.

\section{Authors' contributions}

Experiments were designed by $A B, R D$ and IS. Experiments and analysis were equally conducted by $A B$ and RD. The manuscript was equally prepared and edited by AB, RD and IS. All authors read and approved the final manuscript.

\section{Author's information}

$A B$ and $R D$ are joint first authors.

\section{Acknowledgements}

We thank Jian Qin (Flinders University, Australia) for N. oculata cells, Carlos Gurgel (The University of Adelaide, Australia) for C. taxifolia rhizomes and the Genomics and Bioinformatics core facilities (IMVS ACRF, Adelaide) for Illumina sequencing. We thank Joy Raison and Simon Baxter (The University of Adelaide, Australia) for assistance in generating the circle plots and critical reading of the manuscript respectively. This research was supported by ARC grants DP0988846 and DP110103805 awarded to I.S. and an APA and a GRDC PhD top-up scholarship awarded to AB.

\section{Author details}

'School of Biological Sciences, The University of Adelaide, Adelaide, South Australia 5005, Australia. ${ }^{2}$ School of Agriculture, Food and Wine, The Waite Research Institute, The University of Adelaide, Adelaide, South Australia 5005, Australia. ${ }^{3}$ The University of Adelaide and Shanghai Jiao Tong University Joint International Centre for Agriculture and Health, Adelaide, Australia.

Received: 23 May 2015 Accepted: 24 July 2015

Published online: 14 August 2015

\section{References}

1. Suzuki MM, Bird A. DNA methylation landscapes: provocative insights from epigenomics. Nat Rev Genet. 2008;9:465-76.

2. Motorin Y, Lyko F, Helm M. 5-methylcytosine in RNA: detection, enzymatic formation and biological functions. Nucleic Acids Res. 2010;38:1415-30.

3. Edelheit S, Schwartz S, Mumbach MR, Wurtzel O, Sorek R. Transcriptome-wide mapping of 5-methylcytidine RNA modifications in bacteria, archaea, and yeast reveals $\mathrm{m} 5 \mathrm{C}$ within archaeal mRNAs. PLoS Genet. 2013;9, e1003602.

4. Jackman JE, Alfonzo JD. Transfer RNA modifications: nature's combinatorial chemistry playground. Wires RNA. 2013;4:35-48.

5. Motorin Y, Helm M. tRNA Stabilization by modified nucleotides. Biochemistry. 2010;49:4934-44

6. Alexandrov A, Chernyakov I, Gu WF, Hiley SL, Hughes TR, Grayhack EJ, et al. Rapid tRNA decay can result from lack of nonessential modifications. Mol Cell. 2006;21:87-96.

7. Agris PF. Bringing order to translation: the contributions of transfer RNA anticodon-domain modifications. EMBO Rep. 2008;9:629-35.

8. Helm M. Post-transcriptional nucleotide modification and alternative folding of RNA. Nucleic Acids Res. 2006;34:721-33.

9. Chan CT, Dyavaiah M, DeMott MS, Taghizadeh K, Dedon PC, Begley TJ. A quantitative systems approach reveals dynamic control of tRNA modifications during cellular stress. PLoS Genet. 2010;6, e1001247.

10. Chan CTY, Pang YLJ, Deng WJ, Babu IR, Dyavaiah M, Begley TJ, et al. Reprogramming of tRNA modifications controls the oxidative stress response by codon-biased translation of proteins. Nat Commun. 2012;3.

11. Tuorto F, Liebers R, Musch T, Schaefer M, Hofmann S, Kellner S, et al. RNA cytosine methylation by Dnmt2 and NSun2 promotes tRNA stability and protein synthesis. Nat Struct Mol Biol. 2012;19:900-5.

12. Schaefer M, Pollex T, Hanna K, Tuorto F, Meusburger M, Helm M, et al. RNA methylation by Dnmt2 protects transfer RNAs against stress-induced cleavage. Genes Dev. 2010;24:1590-5.

13. Gigova A, Duggimpudi S, Pollex T, Schaefer M, Kos M. A cluster of methylations in the domain IV of $25 \mathrm{~S}$ rRNA is required for ribosome stability. RNA. 2014;20:1632-44. 
14. Hong B, Brockenbrough JS, Wu P, Aris JP. Nop2p is required for pre-rRNA processing and 60S ribosome subunit synthesis in yeast. Mol Cell Biol. 1997;17:378-88.

15. Sharma S, Yang J, Watzinger $P$, Kotter $P$, Entian KD. Yeast Nop2 and Rcm 1 methylate C2870 and C2278 of the $25 S$ rRNA, respectively. Nucleic Acids Res. 2013;41:9062-76.

16. Motorin Y, Grosjean H. Multisite-specific tRNA : m(5)C-methyltransferase (Trm4) in yeast Saccharomyces cerevisiae: Identification of the gene and substrate specificity of the enzyme. RNA. 1999;5:1105-18.

17. Auxilien S, Guerineau V, Szweykowska-Kulinska Z, Golinelli-Pimpaneau B. The Human tRNA m(5)C methyltransferase Misu is multisite-specific. RNA Biol. 2012;9:1331-8

18. Blanco S, Dietmann S, Flores JV, Hussain S, Kutter C, Humphreys P, et al. Aberrant methylation of tRNAs links cellular stress to neuro-developmental disorders. EMBO J. 2014;33:2020-39.

19. Khan MA, Rafiq MA, Noor A, Hussain S, Flores JV, Rupp V, et al. Mutation in NSUN2, which encodes an RNA methyltransferase, causes autosomal-recessive intellectual disability. Am J Hum Genet. 2012;90:856-63.

20. Abbasi-Moheb L, Mertel S, Gonsior M, Nouri-Vahid L, Kahrizi K, Cirak S, et al. Mutations in NSUN2 cause autosomal-recessive intellectual disability. Am J Hum Genet. 2012;90:847-55.

21. Martinez FJ, Lee JH, Lee JE, Blanco S, Nickerson E, Gabriel S, et al. Whole exome sequencing identifies a splicing mutation in NSUN2 as a cause of a Dubowitz-like syndrome. Journal of Medical Genetics. 2012:49:380-5.

22. Fahiminiya S, Almuriekhi M, Nawaz Z, Staffa A, Lepage P, Ali R, et al. Whole exome sequencing unravels disease-causing genes in consanguineous families in Qatar. Clin Genet. 2014;86:134-41.

23. Blanco S, Kurowski A, Nichols J, Watt FM, Benitah SA, Frye M. The RNA-methyltransferase Misu (NSun2) poises epidermal stem cells to differentiate. PLoS Genet. 2011;7, e1002403.

24. Hussain S, Tuorto F, Menon S, Blanco S, Cox C, Flores JV, et al. The mouse cytosine-5 RNA methyltransferase NSun2 is a component of the chromatoid body and required for testis differentiation. Mol Cell Biol. 2013;33:1561-70.

25. Pavlopoulou A, Kossida S. Phylogenetic analysis of the eukaryotic RNA (cytosine-5)-methyltransferases. Genomics. 2009;93:350-7.

26. Chen $P$, Jager $G$, Zheng B. Transfer RNA modifications and genes for modifying enzymes in Arabidopsis thaliana. BMC Plant Biol. 2010;10:201.

27. Goll MG, Kirpekar F, Maggert KA, Yoder JA, Hsieh CL, Zhang XY, et al. Methylation of tRNA(AsP) by the DNA methyltransferase homolog Dnmt2. Science. 2006;311:395-8.

28. Rai K, Chidester S, Zavala CV, Manos EJ, James SR, Karpf AR, et al. Dnmt2 functions in the cytoplasm to promote liver, brain, and retina development in zebrafish. Genes Dev. 2007;21:261-6.

29. Squires JE, Patel HR, Nousch M, Sibbritt T, Humphreys DT, Parker BJ, et al. Widespread occurrence of 5-methylcytosine in human coding and non-coding RNA. Nucleic Acids Res. 2012;40:5023-33.

30. Khoddami V, Cairns BR. Identification of direct targets and modified bases of RNA cytosine methyltransferases. Nat Biotechnol. 2013;31:458-64.

31. Hussain S, Sajini AA, Blanco S, Dietmann S, Lombard P, Sugimoto Y, et al. NSun2-mediated cytosine-5 methylation of vault noncoding RNA determines its processing into regulatory small RNAs. Cell Rep. 2013;4:255-61.

32. Suzuki T, Suzuki T. A complete landscape of post-transcriptional modifications in mammalian mitochondrial tRNAs. Nucleic Acids Res. 2014;42:7346-57.

33. Sugiura M, Hirose T, Sugita M. Evolution and mechanism of translation in chloroplasts. Annu Rev Genet. 1998;32:437-59.

34. Granneman S, Baserga SJ. Ribosome biogenesis: of knobs and RNA processing. Exp Cell Res. 2004:296:43-50.

35. Lindahl L, Zengel JM. Ribosomal genes in Escherichia coli. Annu Rev Genet. 1986:20:297-326.

36. Maden BE. Locations of methyl groups in $28 \mathrm{~S}$ rRNA of Xenopus laevis and man Clustering in the conserved core of molecule. J Mol Biol. 1988;201:289-314.

37. Purta E, O'Connor M, Bujnicki JM, Douthwaite S. YccW is the m5C methyltransferase specific for 235 rRNA nucleotide 1962. J Mol Biol. 2008;383:641-51

38. Gu XR, Gustafsson C, Ku J, Yu M, Santi DV. Identification of the 16S rRNA m5C967 methyltransferase from Escherichia coli. Biochemistry. 1999;38:4053-7.

39. Andersen NM, Douthwaite S. YebU is a $\mathrm{m} 5 \mathrm{C}$ methyltransferase specific for 16 S rRNA nucleotide 1407. J Mol Biol. 2006;359:777-86.

40. Baer RJ, Dubin DT. Methylated regions of hamster mitochondrial ribosomal RNA: structural and functional correlates. Nucleic Acids Res. 1981;9:323-37.

41. Metodiev MD, Spahr H, Loguercio Polosa P, Meharg C, Becker C, Altmueller J, et al. NSUN4 is a dual function mitochondrial protein required for both methylation of 125 rRNA and coordination of mitoribosomal assembly. PLoS Genet. 2014;10, e1004110.

42. Fujikura U, Horiguchi G, Ponce MR, Micol JL, Tsukaya H. Coordination of cell proliferation and cell expansion mediated by ribosome-related processes in the leaves of Arabidopsis thaliana. Plant J. 2009;59:499-508.

43. Hebrard C, Trap-Gentil MV, Lafon-Placette C, Delaunay A, Joseph C, Lefebvre $M$, et al. Identification of differentially methylated regions during vernalization revealed a role for RNA methyltransferases in bolting. J Exp Bot. 2013;64:651-63.

44. Duchene AM, Pujol C, Marechal-Drouard L. Import of tRNAs and aminoacyl-tRNA synthetases into mitochondria. Curr Genet. 2009;55:1-18.

45. Marechaldrouard L, Weil JH, Dietrich A. Transfer-Rnas and Transfer-Rna Genes in Plants. Annu Rev Plant Phys. 1993;44:13-32.

46. Adams KL, Palmer JD. Evolution of mitochondrial gene content: gene loss and transfer to the nucleus. Mol Phylogenet Evol. 2003;29:380-95.

47. Michaud M, Cognat V, Duchene AM, Marechal-Drouard L. A global picture of tRNA genes in plant genomes. Plant J. 2011;66:80-93.

48. Yukawa Y, Mizutani T, Akama K, Sugiura M. A survey of expressed tRNA genes in the chromosome I of Arabidopsis using an RNA polymerase III-dependent in vitro transcription system. Gene. 2007;392:7-13.

49. Cognat V, Pawlak G, Duchene AM, Daujat M, Gigant A, Salinas T, et al. PlantRNA, a database for tRNAs of photosynthetic eukaryotes. Nucleic Acids Res. 2013;41:D273-279.

50. Zhou W, Karcher D, Fischer A, Maximova E, Walther D, Bock R. Multiple RNA processing defects and impaired chloroplast function in plants deficient in the organellar protein-only RNase P enzyme. PLoS One. 2015;10, e0120533.

51. Schaefer M, Pollex T, Hanna K, Lyko F. RNA cytosine methylation analysis by bisulfite sequencing. Nucleic Acids Res. 2009;37, e12.

52. Bujnicki JM, Feder M, Ayres CL, Redman KL. Sequence-structure-function studies of tRNA : $m(5) C$ methyltransferase Trm4p and its relationship to DNA : m(5)C and RNA : m(5)U methyltransferases. Nucleic Acids Res. 2004;32:2453-63.

53. Borovinskaya MA, Shoji S, Fredrick K, Cate JHD. Structural basis for hygromycin B inhibition of protein biosynthesis. RNA. 2008;14:1590-9.

54. King MY, Redman KL. RNA methyltransferases utilize two cysteine residues in the formation of 5-methylcytosine. Biochemistry. 2002;41:11218-25.

55. Liu YQ. Santi DV: $m(5) C$ RNA and $m(5) C$ DNA methyl transferases use different cysteine residues as catalysts. Proc Natl Acad Sci. 2000;97:8263-5.

56. Foster PG, Nunes CR, Greene P, Moustakas D, Stroud RM. The first structure of an RNA m(5)C methyltransferase, Fmu, provides insight into catalytic mechanism and specific binding of RNA substrate. Structure. 2003;11:1609-20.

57. Zhang SD, Sun L, Kragler F. The phloem-delivered RNA pool contains small noncoding RNAs and interferes with translation. Plant Physiol. 2009;150:378-87.

58. Loss-Morais G, Waterhouse PM, Margis R. Description of plant tRNA-derived RNA fragments (tRFs) associated with argonaute and identification of their putative targets. Biol Direct. 2013;8.

59. Haussecker D, Huang Y, Lau A, Parameswaran P, Fire AZ, Kay MA. Human tRNA-derived small RNAs in the global regulation of RNA silencing. RNA. 2010;16:673-95.

60. Burroughs AM, Ando Y, de Hoon MJ, Tomaru Y, Suzuki H, Hayashizaki Y, et al. Deep-sequencing of human Argonaute-associated small RNAs provides insight into miRNA sorting and reveals Argonaute association with RNA fragments of diverse origin. RNA Biol. 2011;8:158-77.

61. Sobala A, Hutvagner G. Small RNAs derived from the 5 end of tRNA can inhibit protein translation in human cells. RNA Biol. 2013;10:553-63.

62. Gray MW, Burger G, Lang BF. Mitochondrial evolution. Science. 1999;283:1476-81.

63. Rodriguez-Ezpeleta N, Brinkmann H, Burey SC, Roure B, Burger G, Loffelhardt W, et al. Monophyly of primary photosynthetic eukaryotes: green plants, red algae, and glaucophytes. Curr Biol. 2005;15:1325-30.

64. Suzuki T, Nagao A, Suzuki T. Human mitochondrial tRNAs: biogenesis, function, structural aspects, and diseases. Annu Rev Genet. 2011;45:299-329.

65. Wu P, Brockenbrough JS, Paddy MR, Aris JP. NCL1, a novel gene for a non-essential nuclear protein in Saccharomyces cerevisiae. Gene. 1998;220:109-17.

66. Merret R, Nagarajan VK, Carpentier MC, Park S, Favory JJ, Descombin J, et al. Heat-induced ribosome pausing triggers mRNA co-translational decay in Arabidopsis thaliana. Nucleic Acids Res. 2015;43:4121-32.

67. King M, Ton D, Redman KL. A conserved motif in the yeast nucleolar protein Nop2p contains an essential cysteine residue. Biochem J. 1999;337(Pt 1):29-35.

68. Lamesch P, Berardini TZ, Li D, Swarbreck D, Wilks C, Sasidharan R, et al. The Arabidopsis Information Resource (TAIR): improved gene annotation and new tools. Nucleic Acids Res. 2012;40:D1202-1210. 
69. Sprinzl M, Horn C, Brown M, loudovitch A, Steinberg S. Compilation of tRNA sequences and sequences of tRNA genes. Nucleic Acids Res. 1998;26:148-53.

70. R Core Team. R: A language and environment for statistical computing. R version 3.1.0 edition. Vienna, Austria: R Foundation for Statistical Computing; 2014.

71. Kolde R. pheatmap: Pretty Heatmaps. R package version 0.7.7 edition; 2013.

72. Goujon M, McWilliam H, Li W, Valentin F, Squizzato S, Paern J, et al. A new bioinformatics analysis tools framework at EMBL-EBI. Nucleic Acids Res. 2010:38:W695-699.

73. Alonso JM, Stepanova AN, Leisse TJ, Kim CJ, Chen HM, Shinn P, et al. Genome-wide Insertional mutagenesis of Arabidopsis thaliana. Science. 2003;5633:653-7.

\section{Submit your next manuscript to BioMed Central} and take full advantage of:

- Convenient online submission

- Thorough peer review

- No space constraints or color figure charges

- Immediate publication on acceptance

- Inclusion in PubMed, CAS, Scopus and Google Scholar

- Research which is freely available for redistribution 